\title{
Asymptotic Periodicity for Strongly Damped Wave Equations
}

\author{
Claudio Cuevas, ${ }^{1}$ Carlos Lizama, ${ }^{2}$ and Herme Soto ${ }^{3}$ \\ ${ }^{1}$ Departamento de Matemática, Universidade Federal de Pernambuco 50540-740 Recife, PE, Brazil \\ ${ }^{2}$ Departamento de Matemática, Universidad de Santiago de Chile, Casilla 307, Correo 2, Santiago, Chile \\ ${ }^{3}$ Departamento de Matemática y Estadística, Universidad de La Frontera, Casilla 54-D, Temuco, Chile \\ Correspondence should be addressed to Carlos Lizama; carlos.lizama@usach.cl
}

Received 22 April 2013; Accepted 23 June 2013

Academic Editor: Nasser-Eddine Tatar

Copyright (C) 2013 Claudio Cuevas et al. This is an open access article distributed under the Creative Commons Attribution License, which permits unrestricted use, distribution, and reproduction in any medium, provided the original work is properly cited.

This work deals with the existence and uniqueness of asymptotically almost-periodic mild solutions for a class of strongly damped semilinear wave equations.

\section{Introduction}

Let $X$ be a reflexive Banach space and let $A: D(A) \subseteq X \rightarrow X$ be a closed densely defined operator and $\eta>0$. Consider the Cauchy problem

$$
\begin{gathered}
u_{t t}+2 \eta A^{1 / 2} u_{t}+A u=f\left(t, u, u_{t}\right), \quad t>0, \\
u(0)=u_{0} \in X^{1 / 2}, \quad u_{t}(0)=v_{0} \in X,
\end{gathered}
$$

where $X^{1 / 2}$ is the fractional power space associated with $A$ as in [1]. Equations like (1) appear in the literature under the name of strongly damped wave equations. An example of mathematical model represented in form (1) is the wave equation with structural damping (see [2-5]). The strongly damped wave equations has been investigated in several contexts by many authors in the last years, for example, existence $[6,7]$, global classical solution $[6,8]$, long-time asymptotic behavior [9-11], attractor [2, 12-16], well-posedness [17], decay estimates [18], blowup [8, 19, 20], controllability [19], bootstrapping, and regularity [21]. Another important aspect of the qualitative study of the solutions of strongly damped wave equations is their asymptotic periodicity. In recent years, the study of periodicity and its various extensions for evolution equations has attracted a great deal of attention of many mathematicians (see [22-32] and references therein).

To the best of our knowledge, the study of the existence of asymptotically almost-periodic solutions for strongly damped wave equations of type (1) is a topic not yet considered in the literature.
Problem (1) can be written as a first order in time Cauchy problem in $Y^{0}:=X^{1 / 2} \times X$ :

$$
\begin{gathered}
{\left[\begin{array}{l}
u \\
v
\end{array}\right]_{t}+\mathscr{A}_{(1 / 2)}\left[\begin{array}{c}
u \\
v
\end{array}\right]=F\left(t,\left[\begin{array}{c}
u \\
v
\end{array}\right]\right), \quad t>0,} \\
{\left[\begin{array}{l}
u(0) \\
v(0)
\end{array}\right]=\left[\begin{array}{c}
u_{0} \\
v_{0}
\end{array}\right],}
\end{gathered}
$$

where

$$
\mathscr{A}_{(1 / 2)}=\left[\begin{array}{cc}
0 & -I \\
A & 2 \eta A^{1 / 2}
\end{array}\right]: D\left(\mathscr{A}_{(1 / 2)}\right) \subseteq X^{1 / 2} \times X \rightarrow X^{1 / 2} \times X
$$

is defined by

$$
\begin{gathered}
\mathscr{A}_{(1 / 2)}\left[\begin{array}{l}
\varphi \\
\psi
\end{array}\right]=\left[\begin{array}{c}
-\psi \\
A \varphi+2 \eta A^{1 / 2} \psi
\end{array}\right] \\
\text { for }\left[\begin{array}{l}
\varphi \\
\psi
\end{array}\right] \in D\left(\mathscr{A}_{(1 / 2)}\right)=X^{1} \times X^{1 / 2}, \\
F\left(t,\left[\begin{array}{l}
u \\
v
\end{array}\right]\right)=\left[\begin{array}{c}
0 \\
f(t, u, v)
\end{array}\right] .
\end{gathered}
$$

Definition 1. The pair $(\eta, A)$ is said to be an admissible pair if there exist $\psi \in(0, \pi / 2)$ and $M>0$ such that

$$
\left\|(\lambda I-A)^{-1}\right\|_{\mathscr{L}(X)} \leq \frac{M}{1+|\lambda|}
$$


for all $\lambda$ in the sector $\Sigma_{\psi}=\{\lambda \in \mathbb{C}: \psi \leq|\arg \lambda| \leq \pi\} \cup\{0\}$ and $\pi / 2>\psi / 2+\arg \left(\eta+\sqrt{\eta^{2}-1}\right)$.

If $(\eta, A)$ is an admissible pair, by [21, Proposition 2.1] $\mathscr{A}_{(1 / 2)}$ is a closed operator with $0 \in \rho\left(\mathscr{A}_{(1 / 2)}\right)$. Indeed, $\mathscr{A}_{(1 / 2)}$ has the inverse expressed in the matrix form as

$$
\mathscr{A}_{(1 / 2)}^{-1}=\left[\begin{array}{cc}
2 \eta A^{-1 / 2} & A^{-1} \\
-I & 0
\end{array}\right] \in \mathscr{L}\left(X^{1 / 2} \times X\right) .
$$

Moreover, the operator $\mathscr{A}_{(1 / 2)}$ has compact resolvent whenever $A$ has compact resolvent. By [21, Theorem 2.3] the operator $\mathscr{A}_{(1 / 2)}$ is sectorial in $X^{1 / 2} \times X$. The semigroup $\left\{e^{-\mathscr{A}_{(1 / 2)} t}: \quad t \geq 0\right\}$ generated by $-\mathscr{A}_{(1 / 2)}$ in $X^{1 / 2} \times X$ is exponentially decaying analytic. That is, there are constants $K \geq 1$ and $C>0$ such that

$$
\left\|e^{-\mathscr{A}_{(1 / 2)} t}\right\|_{\mathscr{L}\left(X^{1 / 2} \times X\right)} \leq K e^{-C t}, \quad t \geq 0 .
$$

Throughout this paper, we always assume that $(\eta, A)$ is an admissible pair.

This paper has four sections. In the next section, we consider some definitions, technical aspects, and basic properties related to asymptotically almost-periodic functions. In the third section, we obtain general results on the existence of asymptotically almost-periodic (mild) solutions to the problem (1). The main abstract results are Theorems 12, 16, 17, and 19. Finally, in the fourth section we consider several applications. In particular, we consider the following class of partial differential equations:

$$
\begin{aligned}
& u_{t t}+v b(t) u_{t}+\Delta^{2} u-\delta \Delta u_{t} \\
& =\mu a(t)(h(u) \nabla \cdot u+g(u) \Delta u), \quad x \in \Omega, t \geq 0 \\
& u=\Delta u=0, \quad x \in \partial \Omega, \quad t>0, \\
& u(0, x)=u_{0}(x), \quad u_{t}(0, x)=v_{0}(x), \quad x \in \Omega,
\end{aligned}
$$

in a bounded smooth domain $\Omega \subseteq \mathbb{R}^{N}$ and where $h$ and $g$ satisfy certain growth conditions. We prove that if $a \in$ $A P(\mathbb{R}), b \in C_{0}\left(\mathbb{R}^{+}, \mathbb{R}\right)$, and $|\mu|+|\nu|$ is small enough, then the previous problem has an asymptotically almost periodic mild solution. The same type of conclusion is derived for the wide class

$$
\begin{gathered}
u_{t t}+\Delta^{2} u-\delta \Delta u_{t}=a(t)\left|\int_{\Omega} \nabla \cdot u(t, x) d x\right|^{\beta} \Phi_{0}, \\
x \in \Omega, \quad t \geq 0, \\
u=\Delta u=0, \quad x \in \partial \Omega, \quad t>0, \\
u(0, x)=u_{0}(x), \quad u_{t}(0, x)=v_{0}(x), \quad x \in \Omega,
\end{gathered}
$$

where $\Phi_{0} \in L^{p}(\Omega)$ and $p>N / 2$.

\section{Preliminaries}

In this section, we present some concepts and properties needed to develop the following sections. Let $X$ be a reflexive
Banach space. For an interval $I \subseteq \mathbb{R}, C_{b}(I, X)$ denotes the space formed by the bounded continuous functions from $I$ into $X$, endowed with the norm of uniform convergence. When $X=\mathbb{R}$, we denote $C_{b}(I)$ instead of $C_{b}(I, \mathbb{R})$. The notation $C_{0}\left(\mathbb{R}^{+}, X\right)$ stands for the subspace of $C_{b}\left(\mathbb{R}^{+}, X\right)$ consisting of functions that vanish at infinity. We denote by $\mathscr{L}(X)$ the Banach algebra of bounded linear operators defined on $X$. For $r>0$, the notation $B_{r}(X)$ stands for the closed ball $\{x \in X:\|x\| \leq r\}$. For a linear operator $A$ with domain $D(A)$ and range $\mathscr{R}(A)$ in $X$, we represent by $\sigma(A)$ (resp., $\rho(A))$ the spectrum (resp., the resolvent set) of $A$. For $\lambda \in \rho(A)$, we denote by $R(\lambda, A)=(\lambda I-A)^{-1}$ the resolvent operator of $A$. When $A$ is closed, we denote by $[D(A)]$ the domain of $A$ endowed with the graph norm $\|x\|_{A}=\|x\|+\|A x\|$.

Next, we present a brief summary of the main properties of asymptotically almost-periodic functions.

Definition 2 (see [33]). A continuous function $f: \mathbb{R} \rightarrow X$ is called almost-periodic if for each $\varepsilon>0$ there exists $l(\varepsilon)>0$ such that for every interval of length $l(\varepsilon)$ it contains a number $\tau$ with the property that $\|f(t+\tau)-f(t)\| \leq \varepsilon$ for each $t \in \mathbb{R}$.

The previous number $\tau$ is called an $\varepsilon$-translation number for $f$. We denote by $A P(X)$ the space formed by the almost periodic functions $f: \mathbb{R}^{+} \rightarrow X$. We note that each almostperiodic function is bounded and uniformly continuous. It is well known that the range $\mathscr{R}(f)=\{f(t): t \in \mathbb{R}\}$ of an almost periodic function $f$ is relatively compact. The space $A P(X)$ is a Banach space endowed with the norm of uniform convergence.

Let $Y$ be a Banach space. We have the following concept of parameter-dependent almost-periodic function.

Definition 3. A continuous function $f: \mathbb{R} \times Y \rightarrow X$ is called almost-periodic in $t$ uniformly for $y$ in compact subsets of $Y$ if for every compact subset $K$ of $Y$ and each $\varepsilon>0$ there exists $l(\varepsilon)>0$ such that every interval $I$ of length $l(\varepsilon)$ contains a number $\tau$ with the property that $\|f(t+\tau, y)-f(t, y)\| \leq \varepsilon$ for all $t \in \mathbb{R}, y \in K$.

Henceforth, we abbreviate the terminology by calling almost-periodic from $\mathbb{R} \times Y$ into $X$ to those functions that are almost-periodic in $t$ uniformly for $y$ in compact subsets of $Y$, and we denote by $A P(\mathbb{R} \times Y, X)$ the set formed by the almost-periodic functions from $\mathbb{R} \times Y$ into $X$.

The proof of the following result is similar to the proof of [22, Lemma 2.12] and therefore omitted.

Lemma 4. If $h: \mathbb{R} \rightarrow X^{1 / 2} \times X$ is an almost-periodic function and

$$
u(t)=\int_{-\infty}^{t} e^{-\mathscr{A}_{(1 / 2)}(t-s)} h(s) d s, \quad t \in \mathbb{R},
$$

then $u$ is almost-periodic.

It is well known that the study of composition of two functions with special properties is important and basic for deep investigations. The following result has been established in [34]. 
Lemma 5. Let $f: \mathbb{R} \times Y \rightarrow X$ be an almost-periodic function and let $u: \mathbb{R} \rightarrow Y$ be an almost-periodic function. Then the function $\mathbb{R} \rightarrow X, t \rightarrow f(t, u(t))$ is almost-periodic.

We will need the following definition.

Definition 6. A continuous function $f: \mathbb{R}^{+} \rightarrow X$ is called asymptotically almost-periodic if there exist two functions $f_{a p} \in A P(X)$ and $\varphi_{f} \in C_{0}\left(\mathbb{R}^{+}, X\right)$ such that

$$
f(t)=f_{a p}(t)+\varphi_{f}(t), \quad t \in \mathbb{R}^{+} .
$$

The function $f_{a p}$ is called the almost-periodic part of $f$. We denote by $A A P(X)$ the space formed by the asymptotically almost-periodic functions $f: \mathbb{R}^{+} \rightarrow X$. The space $A A P(X)$ is a Banach space endowed with the norm of uniform convergence. Furthermore, $A A P(X)=A P(X) \oplus C_{0}\left(\mathbb{R}^{+}, X\right)$.

In what follows $C_{0}\left(\mathbb{R}^{+} \times Y, X\right)$ denotes the space consisting of continuous functions $f: \mathbb{R}^{+} \times Y \rightarrow X$ such that $\lim _{t \rightarrow \infty} f(t, y)=0$ uniformly for $y$ in compact subsets of $Y$.

Definition 7. A continuous function $f: \mathbb{R}^{+} \times Y \rightarrow$ $X$ is called asymptotically almost-periodic if there are two functions $g \in A P(\mathbb{R} \times Y, X)$ and $\varphi \in C_{0}\left(\mathbb{R}^{+} \times Y, X\right)$ such that

$$
f(t, y)=g(t, y)+\varphi(t, y), \quad y \in Y, \quad t \in \mathbb{R}^{+} .
$$

We denote by $A A P\left(\mathbb{R}^{+} \times Y, X\right)$ the set consisting of all asymptotically almost-periodic functions from $\mathbb{R}^{+} \times Y$ into $X$.

Let $I \subseteq \mathbb{R}$ be an interval. We have the following concept of function uniformly continuous on compacts sets.

Definition 8. A continuous function $f: I \times Y \rightarrow X$ is called uniformly continuous on compact sets if for all compact set $K \subseteq Y$ and all $\varepsilon>0$ there is $\delta_{\varepsilon, K}>0$ such that $\| f\left(t, y_{1}\right)-$ $f\left(t, y_{2}\right) \| \leq \varepsilon$ for all $t \in I$ and $y_{1}, y_{2} \in K$ with $\left\|y_{1}-y_{2}\right\| \leq \delta_{\varepsilon, K}$.

Lemma 9 (see [22]). Let $f: \mathbb{R}^{+} \times Y \rightarrow X$ be an asymptotically almost-periodic and uniformly continuous on compact sets function. Let $u: \mathbb{R}^{+} \rightarrow Y$ be an asymptotically almost-periodic function. Then the function $\mathbb{R}^{+} \rightarrow X, t \rightarrow$ $f(t, u(t))$ is asymptotically almost-periodic.

The proof of the following result is similar to the proof of [22, Lemma 2.13]. Therefore, we will omit it.

Lemma 10. If $h: \mathbb{R}^{+} \rightarrow X^{1 / 2} \times X$ is an asymptotically almost-periodic function and

$$
u(t)=\int_{0}^{t} e^{-\mathscr{A}_{(1 / 2)}(t-s)} h(s) d s, \quad t \geq 0 .
$$

Then, $u$ is asymptotically almost-periodic.

\section{Asymptotically Almost-Periodic Mild Solutions}

We recall the following definition that will be essential for us.
Definition 11. Let $\left[\begin{array}{l}u_{0} \\ v_{0}\end{array}\right]$ be in $X^{1 / 2} \times X$. We say that $\left[\begin{array}{c}u(\cdot) \\ v(\cdot)\end{array}\right]$ : $\mathbb{R}^{+} \rightarrow X^{1 / 2} \times X$ is a mild solution to (2) (or to (1)) if it satisfies the Cauchy integral formula:

$$
\left[\begin{array}{l}
u(t) \\
v(t)
\end{array}\right]=e^{-\mathscr{A}_{(1 / 2)} t}\left[\begin{array}{l}
u_{0} \\
v_{0}
\end{array}\right]+\int_{0}^{t} e^{-\mathscr{A}_{(1 / 2)}(t-s)} F\left(s,\left[\begin{array}{c}
u(s) \\
v(s)
\end{array}\right]\right) d s,
$$$$
t \geq 0 \text {. }
$$

Theorem 12. Let $f: \mathbb{R}^{+} \times X^{1 / 2} \times X \rightarrow X$ be an asymptotically almost-periodic function and assume that there exists a locally integrable function $L_{f}: \mathbb{R} \rightarrow \mathbb{R}$ satisfying

$$
\begin{aligned}
& \left\|f\left(t, u_{1}, v_{1}\right)-f\left(t, u_{2}, v_{2}\right)\right\|_{X} \\
& \quad \leq L_{f}(t)\left[\left\|u_{1}-u_{2}\right\|_{X^{1 / 2}}+\left\|v_{1}-v_{2}\right\|_{X}\right],
\end{aligned}
$$

for all $\left[\begin{array}{c}u_{i} \\ v_{i}\end{array}\right] \in X^{1 / 2} \times X, i=1,2$ and each $t \geq 0$. If

$$
K \sup _{t \geq 0} \int_{0}^{t} e^{-C(t-s)} L_{f}(s) d s<1
$$

where $K$ and $C$ are given in (7). Then, (2) has a unique asymptotically almost-periodic mild solution.

Proof. We define the map $\mathfrak{F}$ on the space $A A P\left(X^{1 / 2} \times X\right)$ by the expression

$$
\begin{aligned}
\mathfrak{F}\left(\left[\begin{array}{l}
u \\
v
\end{array}\right]\right)(t)= & e^{-\mathscr{A}_{(1 / 2)} t}\left[\begin{array}{c}
u_{0} \\
v_{0}
\end{array}\right] \\
& +\int_{0}^{t} e^{-\mathscr{A}_{(1 / 2)}(t-s)} F\left(s,\left[\begin{array}{c}
u(s) \\
v(s)
\end{array}\right]\right) d s, \quad t \in \mathbb{R}^{+},
\end{aligned}
$$

where

$$
\left[\begin{array}{l}
u(\cdot) \\
v(\cdot)
\end{array}\right]=\left[\begin{array}{l}
u_{a p}(\cdot) \\
v_{a p}(\cdot)
\end{array}\right]+\left[\begin{array}{l}
\varphi_{u}(\cdot) \\
\varphi_{v}(\cdot)
\end{array}\right]
$$

is an asymptotically almost-periodic function; that is,

$$
\begin{aligned}
& {\left[\begin{array}{l}
u_{a p}(\cdot) \\
v_{a p}(\cdot)
\end{array}\right] \in A P\left(X^{1 / 2} \times X\right),} \\
& {\left[\begin{array}{l}
\varphi_{u}(\cdot) \\
\varphi_{v}(\cdot)
\end{array}\right] \in C_{0}\left(\mathbb{R}^{+}, X^{1 / 2} \times X\right) .}
\end{aligned}
$$

Since $f \in A A P\left(\mathbb{R}^{+} \times X^{1 / 2} \times X, X\right)$; then there are two functions $\Phi \in A P\left(\mathbb{R}^{+} \times X^{1 / 2} \times X, X\right)$ and $\Psi \in C_{0}\left(\mathbb{R}^{+} \times\right.$ $\left.X^{1 / 2} \times X, X\right)$ so that $f=\Phi+\Psi$.

We set for $\left[\begin{array}{l}a \\ b\end{array}\right] \in X^{1 / 2} \times X$

$$
\widetilde{\Phi}\left(s,\left[\begin{array}{l}
a \\
b
\end{array}\right]\right)=\left[\begin{array}{c}
0 \\
\Phi(s, a, b)
\end{array}\right], \quad \widetilde{\Psi}\left(s,\left[\begin{array}{l}
a \\
b
\end{array}\right]\right)=\left[\begin{array}{c}
0 \\
\Psi(s, a, b)
\end{array}\right] .
$$


For $t \in \mathbb{R}^{+}$, we have the following decomposition:

$$
\begin{aligned}
\mathfrak{F}\left(\left[\begin{array}{l}
u \\
v
\end{array}\right]\right)(t) & =\int_{0}^{t} e^{-\mathscr{A}_{(1 / 2)}(t-s)}\left(\widetilde{\Phi}\left(s,\left[\begin{array}{c}
u(s) \\
v(s)
\end{array}\right]\right)-\widetilde{\Phi}\left(s,\left[\begin{array}{c}
u_{a p}(s) \\
v_{a p}(s)
\end{array}\right]\right)\right) d s \\
& +\int_{-\infty}^{t} e^{-\mathscr{A}_{(1 / 2)}(t-s)} \widetilde{\Phi}\left(s,\left[\begin{array}{c}
u_{a p}(s) \\
v_{a p}(s)
\end{array}\right]\right) d s \\
& -\int_{-\infty}^{0} e^{-\mathscr{A}_{(1 / 2)}(t-s)} \widetilde{\Phi}\left(s,\left[\begin{array}{c}
u_{a p}(s) \\
v_{a p}(s)
\end{array}\right]\right) d s \\
& +e^{-\mathscr{A}_{(1 / 2)} t}\left[\begin{array}{c}
u_{0} \\
v_{0}
\end{array}\right]+\int_{0}^{t} e^{-\mathscr{A}_{(1 / 2)}(t-s)} \widetilde{\Psi}\left(s,\left[\begin{array}{c}
u(s) \\
v(s)
\end{array}\right]\right) d s \\
:= & \mathscr{G}(t)+\mathscr{H}^{(t)},
\end{aligned}
$$

where

$$
\mathscr{G}(t)=\int_{-\infty}^{t} e^{-\mathscr{A}_{(1 / 2)}(t-s)} \widetilde{\Phi}\left(s,\left[\begin{array}{c}
u_{a p}(s) \\
v_{a p}(s)
\end{array}\right]\right) d s
$$

and $\mathscr{H}(t)$ denotes the remained terms of the previous decomposition.

Next, let us show that $\mathscr{H} \in C_{0}\left(\mathbb{R}^{+}, X^{1 / 2} \times X\right)$. By (7) we have that

$$
t \longrightarrow e^{-\mathscr{A}_{(1 / 2)} t}\left[\begin{array}{c}
u_{0} \\
v_{0}
\end{array}\right] \in C_{0}\left(\mathbb{R}^{+}, X^{1 / 2} \times X\right) .
$$

We observe that

$$
\mathscr{B}:=\left\{\left[\begin{array}{l}
u(t) \\
v(t)
\end{array}\right]: t \geq 0\right\}, \quad \mathscr{B}_{a p}:=\left\{\left[\begin{array}{c}
u_{a p}(t) \\
v_{a p}(t)
\end{array}\right]: t \in \mathbb{R}\right\}
$$

are relatively compact in $X^{1 / 2} \times X$. Since $\Psi \in C_{0}\left(\mathbb{R}^{+} \times X^{1 / 2} \times\right.$ $X, X)$, for each $\varepsilon>0$ there exists a constant $T>0$ such that

$$
\|\widetilde{\Psi}(s, z)\|_{X^{1 / 2} \times X} \leq \varepsilon
$$

for all $t \geq 2 T, z \in \mathscr{B}$.

We deduce

$$
\begin{gathered}
\left\|\int_{0}^{t} e^{-\mathscr{A}_{(1 / 2)}(t-s)} \widetilde{\Psi}\left(s,\left[\begin{array}{c}
u(s) \\
v(s)
\end{array}\right]\right) d s\right\|_{X^{1 / 2} \times X} \\
\quad \leq \frac{K}{C} e^{-C t / 2} \sup _{t \in \mathbb{R}^{+}, z \in \mathscr{B}}\|\widetilde{\Psi}(s, z)\|_{X^{1 / 2} \times X}+\frac{K}{C} \varepsilon .
\end{gathered}
$$

On the other hand, since $\Phi \in A P\left(\mathbb{R}^{+} \times X^{1 / 2} \times X, X\right)$ by [35, Appendix] we get

$$
\begin{aligned}
& \left\|\int_{-\infty}^{0} e^{-\mathscr{A}_{(1 / 2)}(t-s)} \widetilde{\Phi}\left(s,\left[\begin{array}{l}
u_{a p}(s) \\
v_{a p}(s)
\end{array}\right]\right) d s\right\|_{X^{1 / 2} \times X} \\
& \quad \leq \frac{K}{C} e^{-C t} \sup _{t \in \mathbb{R}, z \in \mathscr{B}_{a p}}\|\Phi(s, z)\|_{X}, \quad \text { for } t \in \mathbb{R}^{+} .
\end{aligned}
$$

Next, we estimate the first term of (21). For $\varepsilon>0$, we choose $T>0$ big enough so that

$$
\left\|\varphi_{u}(s)\right\|_{X^{1 / 2}}+\left\|\varphi_{v}(s)\right\|_{X} \leq \varepsilon,
$$

for all $s \geq T$, and $\|\widetilde{\Psi}(s, z)\|_{X^{1 / 2} \times X} \leq \varepsilon$ for all $s \geq T$ and all $z \in \mathscr{B} \cup \mathscr{B}_{a p}$. We have the following estimates:

$$
\begin{aligned}
& \| \int_{0}^{t} e^{-\mathscr{A}_{(1 / 2)}(t-s)}\left(\widetilde{\Phi}\left(s,\left[\begin{array}{l}
u(s) \\
v(s)
\end{array}\right]\right)\right. \\
& \left.-\widetilde{\Phi}\left(s,\left[\begin{array}{c}
u_{a p}(s) \\
v_{a p}(s)
\end{array}\right]\right)\right) d s \|_{X^{1 / 2} \times X} \\
& \leq K \int_{0}^{t} e^{-C(t-s)} L_{f}(s)
\end{aligned}
$$

$$
\begin{gathered}
\times\left(\left\|u(s)-u_{a p}(s)\right\|_{X^{1 / 2}}+\left\|v(s)-v_{a p}(s)\right\|_{X}\right) d s \\
+K \int_{0}^{t} e^{-C(t-s)} \| \widetilde{\Psi}\left(s,\left[\begin{array}{c}
u(s) \\
v(s)
\end{array}\right]\right) \\
-\widetilde{\Psi}\left(s,\left[\begin{array}{c}
u_{a p}(s) \\
v_{a p}(s)
\end{array}\right]\right) \|_{X^{1 / 2} \times X} d s
\end{gathered}
$$$$
\leq K \int_{0}^{T} e^{-C(t-s)} L_{f}(s)\left(\left\|\varphi_{u}(s)\right\|_{X^{1 / 2}}+\left\|\varphi_{v}(s)\right\|_{X}\right) d s
$$$$
+K \int_{T}^{t} e^{-C(t-s)} L_{f}(s)\left(\left\|\varphi_{u}(s)\right\|_{X^{1 / 2}}+\left\|\varphi_{v}(s)\right\|_{X}\right) d s
$$$$
+\left(K \int_{0}^{T} e^{-C(t-s)} d s\right)
$$$$
\times\left(\sup _{\xi \in \mathbb{R}^{+}, z \in \mathscr{B}}\|\widetilde{\Psi}(\xi, z)\|_{X^{1 / 2} \times X}\right.
$$$$
\left.+\sup _{\xi \in \mathbb{R}^{+}, z \in \mathscr{B}_{a p}}\|\widetilde{\Psi}(\xi, z)\|_{X^{1 / 2} \times X}\right)
$$

$$
\begin{aligned}
& +K \int_{T}^{t} e^{-C(t-s)} \\
& \quad \times\left(\left\|\widetilde{\Psi}\left(s,\left[\begin{array}{l}
u(s) \\
v(s)
\end{array}\right]\right)\right\|_{X^{1 / 2} \times X}\right.
\end{aligned}
$$$$
\left.+\left\|\widetilde{\Psi}\left(s,\left[\begin{array}{c}
u_{a p}(s) \\
v_{a p}(s)
\end{array}\right]\right)\right\|_{X^{1 / 2} \times X}\right) d s
$$$$
\leq K e^{-C t}\left(\int_{0}^{T} e^{C s} L_{f}(s) d s\right) \sup _{\xi \in \mathbb{R}^{+}}\left\|\left[\begin{array}{c}
\varphi_{u}(\xi) \\
\varphi_{v}(\xi)
\end{array}\right]\right\|_{X^{1 / 2} \times X}+\varepsilon K
$$

$+\frac{K}{C} e^{C T} e^{-C t}$ 


$$
\begin{aligned}
& \quad \times\left(\sup _{\xi \in \mathbb{R}^{+}, z \in \mathscr{B}}\|\widetilde{\Psi}(\xi, z)\|_{X^{1 / 2} \times X}\right. \\
& \left.\quad+\sup _{\xi \in \mathbb{R}^{+}, z \in \mathscr{B}_{a p}}\|\widetilde{\Psi}(\xi, z)\|_{X^{1 / 2} \times X}\right) \\
& +2 \varepsilon \frac{K}{C} .
\end{aligned}
$$

From (23) to (29), we deduce that $\lim _{t \rightarrow \infty} \mathscr{H}(t)=0$; that is, $\mathscr{H} \in C_{0}\left(\mathbb{R}^{+}, X^{1 / 2} \times X\right)$.

Since $\widetilde{\Phi} \in A P\left(\mathbb{R}^{+} \times X^{1 / 2} \times X, X^{1 / 2} \times X\right)$ and $\left[\begin{array}{l}u_{a p} \\ v_{a p}\end{array}\right] \epsilon$ $A P\left(X^{1 / 2} \times X\right)$, we get from Lemma 5 that

$$
\widetilde{\Phi}\left(\cdot,\left[\begin{array}{c}
u_{a p}(\cdot) \\
v_{a p}(\cdot)
\end{array}\right]\right) \in A P\left(X^{1 / 2} \times X\right) .
$$

Now, by Lemma 4 , we obtain that $\mathscr{G} \in A P\left(X^{1 / 2} \times X\right)$, and hence $\mathfrak{F}$ is well defined. It suffices to show that the operator $\mathfrak{F}$ has a unique fixed point in $\operatorname{AAP}\left(X^{1 / 2} \times X\right)$.

For this, we consider that $\left[\begin{array}{l}u_{1} \\ v_{1}\end{array}\right],\left[\begin{array}{l}u_{2} \\ v_{2}\end{array}\right] \in A A P\left(X^{1 / 2} \times X\right)$. We can deduce that

$$
\begin{aligned}
& \left\|\mathfrak{F}\left[\begin{array}{l}
u_{1} \\
v_{1}
\end{array}\right]-\mathfrak{F}\left[\begin{array}{l}
u_{2} \\
v_{2}
\end{array}\right]\right\|_{\infty} \\
& =\sup _{t \geq 0}\left\|\mathfrak{F}\left(\left[\begin{array}{l}
u_{1} \\
v_{1}
\end{array}\right]\right)(t)-\mathfrak{F}\left(\left[\begin{array}{c}
u_{2} \\
v_{2}
\end{array}\right]\right)(t)\right\|_{X^{1 / 2} \times X} \\
& \leq \sup _{t \geq 0} \int_{0}^{t} e^{-C(t-s)} \\
& \times\left\|f\left(s, u_{1}(s), v_{1}(s)\right)-f\left(s, u_{2}(s), v_{2}(s)\right)\right\|_{X} d s \\
& \leq K \sup _{t \geq 0} \int_{0}^{t} e^{-C(t-s)} L_{f}(s) \\
& \times\left[\left\|u_{1}(s)-u_{2}(s)\right\|_{X^{1 / 2}}+\left\|v_{1}(s)-v_{2}(s)\right\|_{X}\right] d s \\
& \leq\left(K \sup _{t \geq 0} \int_{0}^{t} e^{-C(t-s)} L_{f}(s) d s\right)\left\|\left[\begin{array}{l}
u_{1} \\
v_{1}
\end{array}\right]-\left[\begin{array}{c}
u_{2} \\
v_{2}
\end{array}\right]\right\|_{\infty},
\end{aligned}
$$

by the contraction principle, $\mathfrak{F}$ has a unique fixed point in $A A P\left(X^{1 / 2} \times X\right)$. This completes the proof.

Remark 13. We wish to emphasize that condition (15) is optimal in the sense that the function $L_{f}(\cdot)$ is locally integrable. This is the largest possible class of Lipschitz constant $L_{f}(\cdot)$ for which the conclusion of Theorem 12 holds true. However, this condition in $L_{f}(\cdot)$ makes our analysis much more harder, because to prove Theorem 12 we cannot use the standard composition lemma for asymptotically almostperiodic functions (see Lemma 9). To overcome this difficulty we need to use a suitable decomposition for the natural operator associated with the mild solution (see (14) and (21)). In contrast, we note that in the more restrictive case of $L_{f}(\cdot)$ to be an integrable bounded function we can use Lemma 9 directly.
Corollary 14. Let $f: \mathbb{R}^{+} \times X^{1 / 2} \times X \rightarrow X$ be an asymptotically almost-periodic function that satisfies the Lipschitz condition (15) with $L_{f}(\cdot) \equiv$ L. If

$$
\frac{K L}{C}<1
$$

then problem (2) has a unique asymptotically almost-periodic mild solution.

Remark 15. Let $f: \mathbb{R}^{+} \times X^{1 / 2} \times X \rightarrow X$ be an asymptotically almost-periodic function that satisfies the Lipschitz condition (15). We can avoid the condition (32) by using the fixed-point iteration method. Indeed, we consider two cases.

Case 1. $L_{f}(\cdot) \equiv L$. We consider the following space

$$
\begin{aligned}
A A P^{0} & \left(X^{1 / 2} \times X\right) \\
& :=\left\{\left[\begin{array}{l}
u \\
v
\end{array}\right] \in A A P\left(X^{1 / 2} \times X\right): u(0)=0, v(0)=0\right\}
\end{aligned}
$$

endowed with the norm of the uniform convergence. We define the map $\Gamma$ on the space $A A P^{0}\left(X^{1 / 2} \times X\right)$ by

$$
\begin{aligned}
\Gamma\left(\left[\begin{array}{l}
u \\
v
\end{array}\right]\right)(t)=\int_{0}^{t} & e^{-\mathscr{A}_{(1 / 2)}(t-s)} \\
& \times F\left(s,\left[\begin{array}{c}
u(s) \\
v(s)
\end{array}\right]+e^{-\mathscr{A}_{(1 / 2)} s}\left[\begin{array}{l}
u_{0} \\
v_{0}
\end{array}\right]\right) d s .
\end{aligned}
$$

Combining Lemmas 9 and 10, we know that $\Gamma$ is a continuous function from $A A P^{0}\left(X^{1 / 2} \times X\right)$ into $A A P^{0}\left(X^{1 / 2} \times X\right)$. Moreover, for $\left[\begin{array}{c}u_{i} \\ v_{i}\end{array}\right] \in A A P^{0}\left(X^{1 / 2} \times X\right), i=1,2$ we have

$$
\begin{array}{r}
\left\|\Gamma\left(\left[\begin{array}{l}
u_{1} \\
v_{1}
\end{array}\right]\right)(t)-\Gamma\left(\left[\begin{array}{l}
u_{2} \\
v_{2}
\end{array}\right]\right)(t)\right\|_{X^{1 / 2} \times X} \\
\leq K L \int_{0}^{t} e^{-C(t-s)} \sup _{0 \leq \xi \leq s}\left[\left\|u_{1}(\xi)-u_{2}(\xi)\right\|_{X^{1 / 2}}\right. \\
\left.+\left\|v_{1}(\xi)-v_{2}(\xi)\right\|_{X}\right] d s .
\end{array}
$$

With the notation $\Phi(s)=K L e^{-C s}$ and

$$
\alpha(s)=\sup _{0 \leq \xi \leq s}\left[\left\|u_{1}(\xi)-u_{2}(\xi)\right\|_{X^{1 / 2}}+\left\|v_{1}(\xi)-v_{2}(\xi)\right\|_{X}\right],
$$

the previous estimate yields

$$
\left\|\Gamma\left(\left[\begin{array}{l}
u_{1} \\
v_{1}
\end{array}\right]\right)(t)-\Gamma\left(\left[\begin{array}{c}
u_{2} \\
v_{2}
\end{array}\right]\right)(t)\right\|_{X^{1 / 2} \times X} \leq(\Phi * \alpha)(t), \quad t \geq 0 .
$$

Since $\Phi * \alpha$ is a nondecreasing function, proceeding inductively, we can show that

$$
\begin{array}{r}
\left\|\Gamma^{n}\left(\left[\begin{array}{l}
u_{1} \\
v_{1}
\end{array}\right]\right)(t)-\Gamma^{n}\left(\left[\begin{array}{l}
u_{2} \\
v_{2}
\end{array}\right]\right)(t)\right\|_{X^{1 / 2} \times X} \leq\left(\Phi^{* n} * \alpha\right)(t), \\
t \geq 0,
\end{array}
$$

where $\Phi^{* n}$ denotes the $n$-fold convolution of $\Phi$ with itself. 
On the other hand, the map $\mathcal{\delta}: C_{b}\left(\mathbb{R}^{+}\right) \rightarrow C_{b}\left(\mathbb{R}^{+}\right)$, given by

$$
(\mathcal{S} \alpha)(t)=\int_{0}^{t} \Phi(t-s) \alpha(s) d s, \quad t \geq 0,
$$

is a bounded linear map. Moreover, it follows from [36, Theorem 2.3.5] that $\sigma(\mathcal{S})=\{0\}$, which implies that $\left\|\mathcal{S}^{n}\right\|=$ $\left\|\Phi^{* n}\right\|_{1} \rightarrow 0$ as $n \rightarrow \infty$. This shows that $\Gamma^{n}$ is a contraction for $n$ sufficiently large. As a consequence $\Gamma$ has a fixed point $\left[\begin{array}{l}u \\ v\end{array}\right]$ in $A A P^{0}\left(X^{1 / 2} \times X\right)$.

We note that the function

$$
\left[\begin{array}{l}
x(t) \\
y(t)
\end{array}\right]=\left[\begin{array}{l}
u(t) \\
v(t)
\end{array}\right]+e^{-\mathscr{A}_{(1 / 2)} t}\left[\begin{array}{l}
u_{0} \\
v_{0}
\end{array}\right]
$$

is an asymptotically almost-periodic mild solution to problem (2).

Case 2. $L_{f}(\cdot)$ in (15) is an integrable bounded function. By Lemmas 9 and 10 the space $A A P\left(X^{1 / 2} \times X\right)$ is invariant under $\mathfrak{F}$ (see (17)). The fixed point iteration method and the following estimate

$$
\left\|\mathfrak{F}^{n}\left[\begin{array}{l}
u_{1} \\
v_{1}
\end{array}\right]-\mathfrak{F}^{n}\left[\begin{array}{l}
u_{2} \\
v_{2}
\end{array}\right]\right\|_{\infty} \leq \frac{\left(K\left\|L_{f}\right\|_{1}\right)^{n}}{n !}\left\|\left[\begin{array}{l}
u_{1} \\
v_{1}
\end{array}\right]-\left[\begin{array}{c}
u_{2} \\
v_{2}
\end{array}\right]\right\|_{\infty}
$$

are responsible for the fact that $\mathfrak{F}$ has a unique fixed point in $A A P\left(X^{1 / 2} \times X\right)$. This concludes the discussion of Remark 15 .

We have the following results of the existence of local type.

Theorem 16. Let $f: \mathbb{R}^{+} \times X^{1 / 2} \times X \rightarrow X$ be an asymptotically almost-periodic function that satisfies the Lipschitz condition

$$
\begin{aligned}
& \left\|f\left(t, u_{1}, v_{1}\right)-f\left(t, u_{2}, v_{2}\right)\right\|_{X} \\
& \quad \leq L_{f}(r)\left[\left\|u_{1}-u_{2}\right\|_{X^{1 / 2}}+\left\|v_{1}-v_{2}\right\|_{X}\right],
\end{aligned}
$$

for each $t \geq 0$ and $\left[\begin{array}{c}u_{i} \\ v_{i}\end{array}\right] \in X^{1 / 2} \times X$ such that $\left\|u_{i}\right\|_{X^{1 / 2}}+\left\|v_{i}\right\|_{X} \leq$ $r, i=1,2$ where $L_{f}: \mathbb{R}^{+} \rightarrow \mathbb{R}^{+}$is nondecreasing continuous function such that $L_{f}(0)=0$ and $f(t, 0,0)=0$ for all $t \geq 0$. Then, there exists $\varepsilon>0$ such that, for each $\left[\begin{array}{c}u_{0} \\ v_{0}\end{array}\right] \in X^{1 / 2} \times X$ satisfying $\left\|u_{0}\right\|_{X^{1 / 2}}+\left\|v_{0}\right\|_{X} \leq \varepsilon$, there is a unique asymptotically almost-periodic mild solution of problem (2).

Proof. We choose $\lambda, r>0$ small enough such that $\lambda<1$ and $K\left(\lambda+(1 / C) L_{f}(r)\right)<1$. Assume that $\left\|u_{0}\right\|_{X^{1 / 2}}+\left\|v_{0}\right\|_{X} \leq \lambda r$. We consider the space

$$
\begin{gathered}
A A P_{r}:=\left\{\left[\begin{array}{l}
u \\
v
\end{array}\right] \in A A P\left(X^{1 / 2} \times X\right): u(0)=u_{0}, v(0)=v_{0},\right. \\
\left.\|u(t)\|_{X^{1 / 2}}+\|v(t)\|_{X} \leq r, t \geq 0\right\},
\end{gathered}
$$

endowed with the norm of the uniform convergence. We consider the map $\mathfrak{F}$ given by (17) on $A A P_{r}$. By Lemma 9

$$
\mathfrak{F}\left(A A P_{r}\right) \subseteq A A P\left(X^{1 / 2} \times X\right)
$$

We next prove that $\mathfrak{F}\left(A A P_{r}\right) \subseteq A A P_{r}$.
In fact, if $\left[\begin{array}{l}u \\ v\end{array}\right] \in A A P_{r}$, we have

$$
\begin{aligned}
&\left\|\mathfrak{F}\left(\left[\begin{array}{c}
u \\
v
\end{array}\right]\right)(t)\right\|_{X^{1 / 2} \times X} \\
& \leq K e^{-C t}\left[\left\|u_{0}\right\|_{X^{1 / 2}}+\left\|v_{0}\right\|_{X}\right] \\
&+K \int_{0}^{t} e^{-C(t-s)} L_{f}(r) \\
& \times\left[\|u(s)\|_{X^{1 / 2}}+\|v(s)\|_{X}\right] d s \\
& \leq K\left(\lambda+\frac{1}{C} L_{f}(r)\right) r \\
& \leq r,
\end{aligned}
$$

which permit us to infer that $\mathfrak{F}\left(A A P_{r}\right) \subseteq A A P_{r}$.

On the other hand, for $\left[\begin{array}{c}u_{i} \\ v_{i}\end{array}\right] \in X^{1 / 2} \times X, i=1,2$, we have that

$$
\begin{aligned}
&\left\|\mathfrak{F}\left(\left[\begin{array}{l}
u_{1} \\
v_{1}
\end{array}\right]\right)(t)-\mathfrak{F}\left(\left[\begin{array}{l}
u_{2} \\
v_{2}
\end{array}\right]\right)(t)\right\|_{X^{1 / 2} \times X} \\
& \leq K L_{f}(r) \int_{0}^{t} e^{-C(t-s)} \\
& \times\left[\left\|u_{1}(s)-u_{2}(s)\right\|_{X^{1 / 2}}+\left\|v_{1}(s)-v_{2}(s)\right\|_{X}\right] d s \\
& \leq \frac{K}{C} L_{f}(r)\left\|\left[\begin{array}{c}
u_{1} \\
v_{1}
\end{array}\right]-\left[\begin{array}{c}
u_{2} \\
v_{2}
\end{array}\right]\right\|_{\infty},
\end{aligned}
$$

which shows that $\mathfrak{F}$ is a contraction from $A A P_{r}$ into itself. Therefore, the assertion holds for $\varepsilon=\lambda r$.

Theorem 17. Let $f \in A A P\left(\mathbb{R}^{+} \times X^{1 / 2} \times X, X\right)$ be a function that satisfies the local Lipschitz condition (42) with $L_{f}(\cdot)$ a nondecreasing function. Assume that there is a constant $r>0$ such that

$$
\begin{aligned}
& \frac{K}{C}\left(L_{f}\left(r+C_{\#}\right)+\frac{1}{r}\left(L_{f}\left(C_{\#}\right) C_{\#}+\sup _{t \geq 0}\|f(t, 0,0)\|\right)\right) \\
& \quad<1,
\end{aligned}
$$

where $C_{\#}=K\left[\left\|u_{0}\right\|_{X^{1 / 2}}+\left\|v_{0}\right\|_{X}\right], K$, and $C$ are the constant given in (7). Then, there is an asymptotically almost-periodic mild solution of problem (2).

Proof. We define the map $\Gamma$ on the closed ball

$$
\begin{gathered}
\mathscr{B}_{r}=\left\{\left[\begin{array}{l}
u \\
v
\end{array}\right] \in A A P^{0}\left(X^{1 / 2} \times X\right):\|u(t)\|_{X^{1 / 2}}+\|v(t)\|_{X} \leq r,\right. \\
t \geq 0\}
\end{gathered}
$$

by means of the expression (34). 
If $\left[\begin{array}{c}u \\ v\end{array}\right] \in \mathscr{B}_{r}$, we have the estimate

$$
\begin{aligned}
& \left\|\Gamma\left(\left[\begin{array}{l}
u \\
v
\end{array}\right]\right)(t)\right\|_{X^{1 / 2} \times X} \\
& \leq\left\|\Gamma\left(\left[\begin{array}{l}
u \\
v
\end{array}\right]\right)(t)-\Gamma\left(\left[\begin{array}{l}
0 \\
0
\end{array}\right]\right)(t)\right\|_{X^{1 / 2} \times X} \\
& +\left\|\Gamma\left(\left[\begin{array}{l}
0 \\
0
\end{array}\right]\right)(t)\right\|_{X^{1 / 2} \times X} \\
& \leq K L_{f}\left(r+C_{\#}\right) \int_{0}^{t} e^{-C(t-s)} \\
& \times\left[\|u(s)\|_{X^{1 / 2}}+\|v(s)\|_{X}\right] d s \\
& +K \int_{0}^{t} e^{-C(t-s)} \\
& \times\left\|F\left(s, e^{-\mathscr{A}_{(1 / 2)} s}\left[\begin{array}{c}
u_{0} \\
v_{0}
\end{array}\right]\right)\right\|_{X^{1 / 2} \times X} d s \\
& \leq \frac{K}{C} L_{f}\left(r+C_{\#}\right) r \\
& +\frac{K}{C}\left(L_{f}\left(C_{\#}\right) C_{\#}+\sup _{t \geq 0}\|f(t, 0,0)\|\right) \\
& \leq \frac{K}{C}\left(L_{f}\left(r+C_{\#}\right)\right. \\
& \left.+\frac{1}{r}\left(L_{f}\left(C_{\#}\right) C_{\#}+\sup _{t \geq 0}\|f(t, 0,0)\|\right)\right) r \\
& \leq r .
\end{aligned}
$$

Moreover, for $\left[\begin{array}{c}u_{i} \\ v_{i}\end{array}\right] \in \mathscr{B}_{r}, i=1,2$, we have

$$
\begin{gathered}
\left\|\Gamma\left(\left[\begin{array}{l}
u_{1} \\
v_{1}
\end{array}\right]\right)(t)-\Gamma\left(\left[\begin{array}{l}
u_{2} \\
v_{2}
\end{array}\right]\right)(t)\right\|_{X^{1 / 2} \times X} \\
\leq K L_{f}\left(r+C_{\#}\right) \int_{0}^{t} e^{-C(t-s)} \\
\times\left[\left\|u_{1}(s)-u_{2}(s)\right\|_{X^{1 / 2}}\right. \\
\left.+\left\|v_{1}(s)-v_{2}(s)\right\|_{X}\right] d s \\
\leq K L_{f}\left(r+C_{\#}\right)\left\|\left[\begin{array}{c}
u_{1} \\
v_{1}
\end{array}\right]-\left[\begin{array}{c}
u_{2} \\
v_{2}
\end{array}\right]\right\|_{\infty} \cdot
\end{gathered}
$$

\section{Using (50), we get that $\Gamma$ is a contraction on $\mathscr{B}_{r}$.}

In many concrete situations, the operator $A$ has compact resolvent, which in turn implies that the semigroup $e^{-\mathscr{A}_{(1 / 2)} t}$ generated by the operator $-\mathscr{A}_{(1 / 2)}$ is compact for $t>0$. To exploit this property of compactness, we need to introduce some preliminaries.

Let $Y$ be an arbitrary Banach space and let $h:[0, \infty) \rightarrow$ $[1, \infty)$ be a nondecreasing continuous function such that $h(t) \rightarrow \infty$ when $t \rightarrow \infty$. In next, we denote by $C_{h}(Y)$ the space

$$
C_{h}(Y)=\left\{u \in C\left(\mathbb{R}^{+}, Y\right): \lim _{t \rightarrow 0} \frac{u(t)}{h(t)}=0\right\}
$$

endowed with the norm

$$
\|u\|_{h}=\sup _{t \geq 0} \frac{\|u(t)\|_{Y}}{h(t)} .
$$

For reference purposes, we state the following property.

Lemma 18 (see [26]). A set $\mathscr{K} \subseteq C_{h}(Y)$ is relatively compact in $C_{h}(Y)$ if the following conditions are fulfilled:

(C1) For all $b>0$, the set $\mathscr{K}_{b}=\left\{\left.u\right|_{[0, b]}: u \in \mathscr{K}\right\}$ is relatively compact in $C([0, b], Y)$.

(C2) $\lim _{t \rightarrow \infty}\left(\|u(t)\|_{Y} / h(t)\right)=0$ uniformly for $u \in \mathscr{K}$.

To establish our next result we introduce the following condition.

$(W)$ There is a continuous nondecreasing function $W_{f}$ : $\mathbb{R}^{+} \rightarrow \mathbb{R}^{+}$such that

$$
\|f(t, u, v)\|_{X} \leq W_{f}\left(\|u\|_{X^{1 / 2}}+\|v\|_{X}\right),
$$

for all $t \geq 0$ and all $\left[\begin{array}{l}u \\ v\end{array}\right] \in X^{1 / 2} \times X$.

We next denote

$$
\beta(L)=\sup _{t \geq 0} \frac{K}{h(t)} \int_{0}^{t} e^{-C(t-s)} W_{f}(L h(s)) d s .
$$

We have the following result.

Theorem 19. Assume that the operator A in (1) has compact resolvent. Suppose, in addition, that the following conditions are fulfilled:

(a) The function $f \in A A P\left(\mathbb{R}^{+} \times X^{1 / 2} \times X, X\right)$ is uniformly continuous on compact sets and satisfies the condition $(W)$.

(b) For each $a \geq 0, r>0$, and $0 \leq \xi \leq a$,

$$
\left(e^{-\mathscr{A}_{(1 / 2)}(a+s-\xi)}-e^{-\mathscr{A}_{(1 / 2)}(a-\xi)}\right) F\left(\xi,\left[\begin{array}{l}
u \\
v
\end{array}\right]\right) \longrightarrow 0
$$

as $s \rightarrow \infty$ uniformly for all $\left[\begin{array}{l}u \\ v\end{array}\right]$ so that $\|u\|_{X^{1 / 2}}+\|v\|_{X} \leq r$.

(c) For each $L>0$,

$$
\lim _{t \rightarrow \infty} \frac{1}{h(t)} \int_{0}^{t} e^{-C(t-s)} W_{f}(L h(s)) d s=0 .
$$

(d) For each $\varepsilon>0$, there is $\delta>0$ such that for every $\left[\begin{array}{c}u_{i} \\ v_{i}\end{array}\right] \epsilon$ $C_{h}\left(X^{1 / 2} \times X\right), i=1,2$ with

$\frac{1}{h(t)}\left[\left\|u_{1}(t)-u_{2}(t)\right\|_{X^{1 / 2}}+\left\|v_{1}(t)-v_{2}(t)\right\|_{X}\right] \leq \delta$,

$$
t \geq 0
$$


implies that

$$
\int_{0}^{t} e^{-C(t-s)}\left\|f\left(s, u_{1}(s), v_{1}(s)\right)-f\left(s, u_{2}(s), v_{2}(s)\right)\right\|_{X} d s \leq \varepsilon,
$$

for each $t \geq 0$.

(e) $\liminf \operatorname{in}_{\xi \rightarrow \infty} \beta(\xi) / \xi<1$.

Then, problem (2) has an asymptotically almost-periodic mild solution.

Proof. Let $C_{h}^{0}\left(X^{1 / 2} \times X\right)$ be the space consisting of the functions $\left[\begin{array}{c}u(\cdot) \\ v(\cdot)\end{array}\right]$ in $C_{h}\left(X^{1 / 2} \times X\right)$ such that $u(0)=0, v(0)=0$. It is clear that $C_{h}^{0}\left(X^{1 / 2} \times X\right)$ is a closed subspace of $C_{h}\left(X^{1 / 2} \times\right.$ $X)$. We define the operator $\Gamma$ on $C_{h}^{0}\left(X^{1 / 2} \times X\right)$ by (34). It follows from conditions (a) and (c) that

$$
\begin{aligned}
\frac{1}{h(t)}\left\|\Gamma\left(\left[\begin{array}{l}
u \\
v
\end{array}\right]\right)(t)\right\|_{X^{1 / 2} \times X} & \\
\leq \frac{K}{h(t)} \int_{0}^{t} e^{-C(t-s)} W_{f}(\operatorname{Lh}(s)) d s & \longrightarrow 0, \\
t & \longrightarrow \infty,
\end{aligned}
$$

where

$$
L=\left\|\left[\begin{array}{c}
u \\
v
\end{array}\right]\right\|_{h}+K\left(\left\|u_{0}\right\|_{X^{1 / 2}}+\left\|v_{0}\right\|_{X}\right)
$$

Thus, we have that $\Gamma: C_{h}^{0}\left(X^{1 / 2} \times X\right) \rightarrow C_{h}^{0}\left(X^{1 / 2} \times X\right)$.

We divide the rest of the proof into several steps.

(i) The map $\Gamma$ is continuous. For each $\varepsilon>0$ there is $\delta>0$ such that for $\left[\begin{array}{c}u_{i} \\ v_{i}\end{array}\right] \in C_{h}^{0}\left(X^{1 / 2} \times X\right), i=1,2$

with

$$
\left\|\left[\begin{array}{c}
u_{1}-u_{2} \\
v_{1}-v_{2}
\end{array}\right]\right\|_{h} \leq \delta
$$

implies that

$$
\frac{1}{h(t)}\left\|\Gamma\left(\left[\begin{array}{l}
u_{1} \\
v_{1}
\end{array}\right]\right)(t)-\Gamma\left(\left[\begin{array}{l}
u_{2} \\
v_{2}
\end{array}\right]\right)(t)\right\|_{X^{1 / 2} \times X} \leq \frac{1}{h(t)} \varepsilon \leq \varepsilon,
$$

which shows the assertion.

(ii) The map $\Gamma$ is completely continuous. We take $r>0$ and we set $V=\Gamma\left(B_{r}\left(C_{h}^{0}\left(X^{1 / 2} \times X\right)\right)\right)$.

For $t \geq 0$, we set

$$
V(t):=\left\{\left[\begin{array}{l}
u(t) \\
v(t)
\end{array}\right]:\left[\begin{array}{l}
u \\
v
\end{array}\right] \in V\right\} .
$$

We first show that $V(t)$ is a relatively compact set in $X^{1 / 2} \times X$ for each $t \geq 0$. It follows from the mean value theorem that $V(t) \in \overline{t c\left(\mathscr{K}_{0}\right)}$, where $c\left(\mathscr{K}_{0}\right)$ denotes the convex hull of

$$
\begin{array}{r}
\mathscr{K}_{0}=\left\{e^{-\mathscr{A}_{(1 / 2)}(t-s)} F\left(s,\left[\begin{array}{c}
u \\
v
\end{array}\right]\right): 0 \leq s \leq t,\right. \\
\left.\|u\|_{X^{1 / 2}}+\|v\|_{X} \leq \rho\right\},
\end{array}
$$

where $\rho=h(t)\left(r+K\left(\left\|u_{0}\right\|_{X^{1 / 2}}+\left\|v_{0}\right\|_{X}\right)\right)$, and $K$ is a constant given in (7).

Since

$$
\begin{gathered}
\sup \left\{\left\|F\left(s,\left[\begin{array}{c}
u \\
v
\end{array}\right]\right)\right\|_{X^{1 / 2} \times X}: 0 \leq s \leq t,\right. \\
\left.\|u\|_{X^{1 / 2}}+\|v\|_{X} \leq \rho\right\} \leq W_{f}(\rho),
\end{gathered}
$$

and taking into account that $e^{-\mathscr{A}_{(1 / 2)} t}$ is compact for $t>0$, we infer that $\mathscr{K}_{0}$ is a relatively compact set in $X^{1 / 2} \times X$ and consequently $V(t)$ is also relatively compact. Let now $b>0$ fixed and $V_{b}$ the set formed by the functions $\left[\begin{array}{c}u \\ v\end{array}\right] \in V$ restricted to the interval $[0, b]$. We affirm that the set $V_{b}$ is equicontinuous.

In fact, if

$$
\left[\begin{array}{l}
u \\
v
\end{array}\right]=\Gamma\left(\left[\begin{array}{c}
\tilde{u} \\
\widetilde{v}
\end{array}\right]\right), \quad \text { with } \quad\left[\begin{array}{c}
\widetilde{u} \\
\widetilde{v}
\end{array}\right] \in B_{r}\left(C_{h}^{0}\left(X^{1 / 2} \times X\right)\right) \text {. }
$$

For $s \geq 0$, we obtain the following estimate:

$$
\begin{aligned}
& \left\|\left[\begin{array}{c}
u(t+s)-u(t) \\
v(t+s)-v(t)
\end{array}\right]\right\|_{X^{1 / 2} \times X} \\
& \leq K \int_{t}^{t+s} e^{-C(t+s-\xi)} W_{f}(\operatorname{Lh}(\xi)) d \xi \\
& \quad+\int_{0}^{t} \|\left(e^{-\mathscr{A}_{(1 / 2)}(t+s-\xi)}-e^{-\mathscr{A}_{(1 / 2)}(t-\xi)}\right) \\
& \quad \times F\left(\xi,\left[\begin{array}{c}
\tilde{u}(\xi) \\
\widetilde{v}(\xi)
\end{array}\right]+e^{-\mathscr{A}_{(1 / 2)} \xi}\left[\begin{array}{c}
u_{0} \\
v_{0}
\end{array}\right]\right) \|_{X^{1 / 2} \times X} d \xi,
\end{aligned}
$$

where $L=r+K\left(\left\|u_{0}\right\|_{X^{1 / 2}}+\left\|v_{0}\right\|_{X}\right)$. It is immediate that the first term on the right hand side converges to zero when $s \rightarrow 0$ and, using condition $(b)$ we obtain the second term on the right-hand side also converges to zero when $s \rightarrow 0$ and the convergence is independent of the function $\left[\begin{array}{c}\widetilde{u}(\cdot) \\ \widetilde{v}(\cdot)\end{array}\right]$.

We now show that

$$
\lim _{t \rightarrow \infty} \frac{1}{h(t)}\left[\begin{array}{l}
u(t) \\
v(t)
\end{array}\right]=0
$$

independent of $\left[\begin{array}{c}\tilde{u} \\ \tilde{v}\end{array}\right] \in B_{r}\left(C_{h}^{0}\left(X^{1 / 2} \times X\right)\right)$. This assertion is a direct consequence of the following estimate and the condition (c) :

$$
\frac{1}{h(t)}\left\|\left[\begin{array}{c}
u(t) \\
v(t)
\end{array}\right]\right\|_{X^{1 / 2} \times X} \leq \frac{K}{h(t)} \int_{0}^{t} e^{-C(t-s)} W_{f}(\operatorname{Lh}(s)) d s .
$$


Combining these assertions with Lemma 18, we get that $V$ is a relatively compact set in $C_{h}^{0}\left(X^{1 / 2} \times X\right)$. Since $r$ was chosen arbitrary, this proves that $\Gamma$ is completely continuous.

(iii) There is $r_{0}>0$ such that $\Gamma\left(B_{r_{0}}\left(C_{h}^{0}\left(X^{1 / 2} \times X\right)\right)\right) \subseteq$ $B_{r_{0}}\left(C_{h}^{0}\left(X^{1 / 2} \times X\right)\right)$. In fact, if we assume that the assertion is false; then for all $r>0$ we can choose $\left[\begin{array}{c}u^{r} \\ v^{r}\end{array}\right] \in B_{r_{0}}\left(C_{h}^{0}\left(X^{1 / 2} \times X\right)\right)$ such that

$$
\frac{1}{h(t)}\left\|\Gamma\left(\left[\begin{array}{c}
u^{r} \\
v^{r}
\end{array}\right]\right)(t)\right\|_{X^{1 / 2} \times X}>r
$$

for all $t \in \mathbb{R}^{+}$. Then

$$
\begin{aligned}
& 1 \leq(1\left.+\frac{K\left(\left\|u_{0}\right\|_{X^{1 / 2}}+\left\|v_{0}\right\|_{X}\right)}{r}\right) \\
& \times \frac{\beta\left(r+K\left(\left\|u_{0}\right\|_{X^{1 / 2}}+\left\|v_{0}\right\|_{X}\right)\right)}{r+K\left(\left\|u_{0}\right\|_{X^{1 / 2}}+\left\|v_{0}\right\|_{X}\right)}, \\
& 1 \leq \liminf _{\xi \rightarrow \infty} \frac{\beta(\xi)}{\xi},
\end{aligned}
$$

which contradicts condition (e) and establishes the assertion.

(iv) If $\left[\begin{array}{c}u \\ v\end{array}\right] \in A A P^{0}\left(X^{1 / 2} \times X\right)$, then the function $\mathbb{R}^{+} \rightarrow$ $X^{1 / 2} \times X$ given by

$$
t \longrightarrow\left[\begin{array}{l}
u(t) \\
v(t)
\end{array}\right]+e^{-\mathscr{A}_{(1 / 2)} t}\left[\begin{array}{l}
u_{0} \\
v_{0}
\end{array}\right]
$$

is in $A A P\left(X^{1 / 2} \times X\right)$. Since $f \in A A P\left(\mathbb{R}^{+} \times X^{1 / 2} \times\right.$ $X, X)$ is uniformly continuous on compact sets, we have from Lemma 9 that

$t \longrightarrow F\left(t,\left[\begin{array}{l}u(t) \\ v(t)\end{array}\right]+e^{-\mathscr{A}_{(1 / 2)} t}\left[\begin{array}{l}u_{0} \\ v_{0}\end{array}\right]\right) \in A A P\left(X^{1 / 2} \times X\right)$.

Applying Lemma 10, we obtain that $\Gamma\left(A A P^{0}\left(X^{1 / 2} \times\right.\right.$ $X) \subseteq \subseteq A P^{0}\left(X^{1 / 2} \times X\right)$. Consequently, combining with (iii) we infer that

$$
\begin{gathered}
\Gamma\left({\overline{B_{r_{0}}\left(C_{h}^{0}\left(X^{1 / 2} \times X\right)\right) \cap A A P^{0}\left(X^{1 / 2} \times X\right)}}^{h}\right) \\
\subseteq{\overline{B_{r_{0}}\left(C_{h}^{0}\left(X^{1 / 2} \times X\right)\right) \cap A A P^{0}\left(X^{1 / 2} \times X\right)}}^{h},
\end{gathered}
$$

where $\overline{\mathcal{S}}^{h}$ denotes the closure of $\mathcal{S}$ in $C_{h}\left(X^{1 / 2} \times X\right)$. Using Schauder fixed point theorem, we deduce that $\Gamma$ has a fixed point $\left[\begin{array}{l}u \\ v\end{array}\right] \in \overline{B_{r_{0}}\left(C_{h}^{0}\left(X^{1 / 2} \times X\right)\right) \cap A A P^{0}\left(X^{1 / 2} \times X\right)}{ }^{h}$.

(v) Finally, we show that $\left[\begin{array}{l}u \\ v\end{array}\right] \in A A P^{0}\left(X^{1 / 2} \times X\right)$. Let $\left(\left[\begin{array}{c}u^{n} \\ v^{n}\end{array}\right]\right)_{n}$ be a sequence in $B_{r_{0}}\left(C_{h}^{0}\left(X^{1 / 2} \times X\right)\right) \cap$ $A A P^{0}\left(X^{1 / 2} \times X\right)$ that converges to $\left[\begin{array}{l}u \\ v\end{array}\right]$ for the topology in $C_{h}\left(X^{1 / 2} \times X\right)$. For $\varepsilon>0$, let $\delta>0$ be the constant in condition $(d)$; there is $n_{0} \in \mathbb{N}$ so that

$$
\frac{1}{h(t)}\left\|\left[\begin{array}{c}
u^{n}(t)-u(t) \\
v^{n}(t)-v(t)
\end{array}\right]\right\|_{X^{1 / 2} \times X} \leq \delta,
$$

for all $t \geq 0$ and all $n \geq n_{0}$. Therefore, for $n \geq n_{0}$

$$
\sup _{t \geq 0}\left\|\Gamma\left(\left[\begin{array}{c}
u^{n} \\
v^{n}
\end{array}\right]\right)(t)-\Gamma\left(\left[\begin{array}{c}
u \\
v
\end{array}\right]\right)(t)\right\|_{X^{1 / 2} \times X} \leq \varepsilon .
$$

Hence

$$
\lim _{n \rightarrow \infty}\left\|\Gamma\left[\begin{array}{l}
u^{n} \\
v^{n}
\end{array}\right]-\left[\begin{array}{l}
u \\
v
\end{array}\right]\right\|_{\infty}=0 .
$$

Since $\Gamma\left[\begin{array}{l}u^{n} \\ v^{n}\end{array}\right] \in A A P^{0}\left(X^{1 / 2} \times X\right)$ we get that $\left[\begin{array}{l}u \\ v\end{array}\right] \in$ $A A P^{0}\left(X^{1 / 2} \times X\right)$ and completes the proof.

Remark 20. Note that in Theorem 19 we do not need to assume that the operator $A$ in (1) has compact resolvent if the following condition holds.

(f) For all $a \geq 0$ and $r>0$ the set $\left\{f\left(t, \varphi_{1}, \varphi_{2}\right): 0 \leq t \leq\right.$ $\left.a,\left\|\varphi_{1}\right\|_{X^{1 / 2}}+\left\|\varphi_{2}\right\|_{X} \leq r\right\}$ is relatively compact in $X$.

\section{Applications}

Suppose that $h, g \in C(\mathbb{R}, \mathbb{R}), a: \mathbb{R} \rightarrow \mathbb{R}\left(b: \mathbb{R}^{+} \rightarrow \mathbb{R}\right)$ is a bounded continuous function, $\nu, \mu \in \mathbb{R}, \delta>0$ and $\rho_{1}, \rho_{2} \in$ $(1,+\infty)$. In a bounded smooth domain $\Omega \subseteq \mathbb{R}^{N}$, we consider the following partial differential equation:

$$
\begin{gathered}
u_{t t}+v b(t) u_{t}+\Delta^{2} u-\delta \Delta u_{t}=\mu a(t)(h(u) \nabla \cdot u+g(u) \Delta u) \\
x \in \Omega, \quad t \geq 0, \\
u=\Delta u=0, \quad x \in \partial \Omega, \quad t>0, \\
u(0, x)=u_{0}(x), \quad u_{t}(0, x)=v_{0}(x), \quad x \in \Omega,
\end{gathered}
$$

where $h$ and $g$ satisfy the following growth conditions:

$$
\begin{array}{r}
\left|h\left(s_{1}\right)-h\left(s_{2}\right)\right| \leq C_{h}\left|s_{1}-s_{2}\right|\left(1+\left|s_{1}\right|^{\rho_{1}-1}+\left|s_{2}\right|^{\rho_{1}-1}\right), \\
s_{1}, s_{2} \in \mathbb{R}, \\
\left|g\left(s_{1}\right)-g\left(s_{2}\right)\right| \leq C_{g}\left|s_{1}-s_{2}\right|\left(1+\left|s_{2}\right|^{\rho_{2}-1}+\left|s_{1}\right|^{\rho_{2}-1}\right), \\
s_{1}, s_{2} \in \mathbb{R},
\end{array}
$$

where $C_{h}$ and $C_{g}$ are positive constants. Here, we describe the asymptotically almost-periodic behavior of solutions of problem (79) in the $L^{p}$-setting. To model this problem in the abstract form (1) we set that $\eta=\delta / 2, p>N / 2$, the operator $A$ is defined in $L^{p}(\Omega)$ by $A u=\Delta_{D}^{2} u\left(\Delta_{D}\right.$ is the Dirichlet Laplacian in $\Omega$ ) on the domain

$$
D\left(\Delta_{D}^{2}\right)=\left\{\phi \in H_{p}^{4}(\Omega): \phi=\Delta \phi=0 \text { on } \partial \Omega\right\},
$$


where $H_{p}^{4}(\Omega)=W^{4, p}(\Omega)$ is the standard Sobolev space (see [37]). With this specification, problem (79) will fall into the abstract formulation (1). Since $A^{1 / 2}=-\Delta_{D}$, we can choose the angle $\psi$ for the sector

$$
\Sigma_{\psi / 2}=\left\{\lambda \in \mathbb{C}: \frac{\psi}{2} \leq|\arg \lambda| \leq \pi \text { with } \psi \in\left(0, \frac{\pi}{2}\right)\right\}
$$

as small as needed and therefor (see [21, Example 4.3]) $(\eta, A)$ will be an admissible pair for any $\eta>0$. From [21, Section 3] we get that

$$
\left[L^{p}(\Omega)\right]^{1 / 2}=\left\{\phi \in H_{p}^{2}(\Omega): \phi=\Delta \phi=0 \text { on } \partial \Omega\right\} .
$$

We define $f: \mathbb{R}^{+} \times\left[L^{p}(\Omega)\right]^{1 / 2} \times L^{p}(\Omega) \rightarrow L^{p}(\Omega)$ by

$$
\begin{aligned}
& f\left(t, \varphi_{1}, \varphi_{2}\right) \\
& =\mu a(t)\left(h^{e}\left(\varphi_{1}\right) \nabla \cdot \varphi_{1}+g^{e}\left(\varphi_{1}\right) \Delta \varphi_{1}\right)-\nu b(t) \varphi_{2}, \\
& \quad t \in \mathbb{R}^{+}, \quad \varphi_{1} \in\left[L^{p}(\Omega)\right]^{1 / 2}, \quad \varphi_{2} \in L^{p}(\Omega),
\end{aligned}
$$

where $\theta^{e}$ is the Nemytskii operator associated with $\theta$, and $\nabla \varphi$ represents the divergence of $\varphi$. For $\varphi_{1} \in\left[L^{p}(\Omega)\right]^{1 / 2}$ and $\varphi_{2} \in L^{p}(\Omega)$. Using Minkowski's inequality and Sobolev embedding, we have the estimate

$$
\begin{aligned}
& \left\|f\left(t, \varphi_{1}, \varphi_{2}\right)\right\|_{L^{p}(\Omega)} \\
& \leq|\mu||a(t)|\left(\left\|h^{e}\left(\varphi_{1}\right)\right\|_{L^{\infty}(\Omega)}+\left\|g^{e}\left(\varphi_{1}\right)\right\|_{L^{\infty}(\Omega)}\right) \\
& \quad \times\left\|\varphi_{1}\right\|_{H_{p}^{2}(\Omega)}+|\nu||b(t)|\left\|\varphi_{2}\right\|_{L^{p}(\Omega)} .
\end{aligned}
$$

Whence $f$ is well defined. We claim that $f$ satisfies (42) with

$$
\begin{aligned}
L_{f}(r)=\widetilde{C} & (|\mu|+|\nu|)\left(\|a\|_{\infty}+\|b\|_{\infty}\right) \\
& \times\left(1+2 r+3\left(r^{\rho_{1}}+r^{\rho_{2}}\right)\right) .
\end{aligned}
$$

Indeed, it is an easy consequence of the following estimates (here $\widetilde{C}$ will stand for some positive constant independent of $\widetilde{\varphi}_{1}$ and $\widetilde{\varphi}_{2}$ with $\left.\widetilde{\varphi}_{1}, \widetilde{\varphi}_{2} \in\left[L^{p}(\Omega)\right]^{1 / 2}\right)$.

$$
\begin{aligned}
& \left\|\left(h^{e}\left(\widetilde{\varphi}_{1}\right)-h^{e}\left(\widetilde{\varphi}_{2}\right)\right) \nabla \cdot \widetilde{\varphi}_{1}\right\|_{L^{p}(\Omega)} \\
& \leq \widetilde{C}\left\|\widetilde{\varphi}_{1}-\widetilde{\varphi}_{2}\right\|_{H_{p}^{2}(\Omega)} \\
& \quad \times\left(1+\left\|\widetilde{\varphi}_{1}\right\|_{H_{p}^{2}(\Omega)}^{\rho_{1}-1}+\left\|\widetilde{\varphi}_{2}\right\|_{H_{p}^{2}(\Omega)}^{\rho_{1}-1}\right)\left\|\widetilde{\varphi}_{1}\right\|_{H_{p}^{2}(\Omega)},
\end{aligned}
$$

$\left\|h^{e}\left(\widetilde{\varphi}_{2}\right) \nabla \cdot\left(\widetilde{\varphi}_{1}-\widetilde{\varphi}_{2}\right)\right\|_{L^{p}(\Omega)}$

$$
\leq \widetilde{C}\left(1+\left\|\widetilde{\varphi}_{2}\right\|_{H_{p}^{2}(\Omega)}+\left\|\widetilde{\varphi}_{2}\right\|_{H_{p}^{2}(\Omega)}^{\rho_{1}}\right)\left\|\widetilde{\varphi}_{1}-\widetilde{\varphi}_{2}\right\|_{H_{p}^{2}(\Omega)},
$$

$\left\|g^{e}\left(\widetilde{\varphi}_{1}\right) \Delta\left(\widetilde{\varphi}_{1}-\widetilde{\varphi}_{2}\right)\right\|_{L^{p}(\Omega)}$

$$
\begin{aligned}
& \quad \leq \widetilde{C}\left(1+\left\|\widetilde{\varphi}_{1}\right\|_{H_{p}^{2}(\Omega)}+\left\|\widetilde{\varphi}_{1}\right\|_{H_{p}^{2}(\Omega)}^{\rho_{2}}\right)\left\|\widetilde{\varphi}_{2}\right\|_{H_{p}^{2}(\Omega)}, \\
& \left\|\left(g^{e}\left(\widetilde{\varphi}_{1}\right)-g^{e}\left(\widetilde{\varphi}_{2}\right)\right) \Delta \widetilde{\varphi}_{2}\right\|_{L^{p}(\Omega)} \\
& \quad \leq \widetilde{C}\left\|\widetilde{\varphi}_{1}-\widetilde{\varphi}_{2}\right\|_{H_{p}^{2}(\Omega)}
\end{aligned}
$$

$$
\times\left(1+\left\|\widetilde{\varphi}_{1}\right\|_{H_{p}^{2}(\Omega)}^{\rho_{2}-1}+\left\|\widetilde{\varphi}_{2}\right\|_{H_{p}^{2}(\Omega)}^{\rho_{2}-1}\right)\left\|\widetilde{\varphi}_{2}\right\|_{H_{p}^{2}(\Omega)} .
$$

Let $K$ be a compact subset of $\left[L^{p}(\Omega)\right]^{1 / 2} \times L^{p}(\Omega)$. For $\varphi_{1} \epsilon$ $\left[L^{p}(\Omega)\right]^{1 / 2}$ and $\varphi_{2} \in L^{p}(\Omega)$, we set that

$$
\begin{aligned}
& f_{a p}\left(t, \varphi_{1}, \varphi_{2}\right)=\mu a(t)\left(h^{e}\left(\varphi_{1}\right) \nabla \cdot \varphi_{1}+g^{e}\left(\varphi_{1}\right) \Delta \varphi_{1}\right), \\
& \Phi_{f}\left(t, \varphi_{1}, \varphi_{2}\right)=\nu b(t) \varphi_{2}, \\
& M_{K}=\sup \left\{\left\|\left[\begin{array}{l}
\varphi_{1} \\
\varphi_{2}
\end{array}\right]\right\|_{\left[L^{p}(\Omega)\right]^{1 / 2} \times L^{p}(\Omega)}:\left[\begin{array}{l}
\varphi_{1} \\
\varphi_{2}
\end{array}\right] \in K\right\} .
\end{aligned}
$$

We have the following estimates:

$$
\begin{gathered}
\left\|\Phi_{f}\left(t, \varphi_{1}, \varphi_{2}\right)\right\|_{L^{p}(\Omega)} \leq|\nu||b(t)| M_{K}, \\
\left\|f_{a p}\left(t+\tau, \varphi_{1}, \varphi_{2}\right)-f_{a p}\left(t, \varphi_{1}, \varphi_{2}\right)\right\|_{L^{p}(\Omega)} \\
\leq|\mu||a(t+\tau)-a(t)| \\
\times\left(\left\|h^{e}\left(\varphi_{1}\right)\right\|_{L^{\infty}(\Omega)}+\left\|g^{e}\left(\varphi_{1}\right)\right\|_{L^{\infty}(\Omega)}\right)\left\|\varphi_{1}\right\|_{H_{p}^{2}(\Omega)} \\
\leq \widetilde{C}|\mu||a(t+\tau)-a(t)| \\
\times\left(1+\left\|\varphi_{1}\right\|_{H_{p}^{2}(\Omega)}+\left\|\varphi_{1}\right\|_{H_{p}^{2}(\Omega)}^{\rho_{1}}+\left\|\varphi_{1}\right\|_{H_{p}^{2}(\Omega)}^{\rho_{2}}\right)\left\|\varphi_{1}\right\|_{H_{p}^{2}(\Omega)} \\
\leq \widetilde{C}(K)|a(t+\tau)-a(t)|,
\end{gathered}
$$

where $\widetilde{C}(K)$ is a constant depending on $K$.

If $b \in C_{0}\left(\mathbb{R}^{+}, \mathbb{R}\right)$ from (89), we get

$$
\Phi_{f} \in C_{0}\left(\mathbb{R}^{+} \times\left[L^{p}(\Omega)\right]^{1 / 2} \times L^{p}(\Omega), L^{p}(\Omega)\right),
$$

and (90) implies that

$$
f_{a p} \in A P\left(\mathbb{R} \times\left[L^{p}(\Omega)\right]^{1 / 2} \times L^{p}(\Omega), L^{p}(\Omega)\right) .
$$


Hence,

$$
f \in A A P\left(\mathbb{R}^{+} \times\left[L^{p}(\Omega)\right]^{1 / 2} \times L^{p}(\Omega), L^{p}(\Omega)\right) .
$$

Applying Theorem 17, we have the following result.

Proposition 21. Under the previous conditions, if a $\epsilon$ $A P(\mathbb{R}), b \in C_{0}\left(\mathbb{R}^{+}, \mathbb{R}\right)$, and $|\mu|+|\nu|$ is small enough, then problem (79) has an asymptotically almost-periodic mild solution.

Example 22. We consider the following partial differential equation

$$
\begin{gathered}
u_{t t}+\frac{v}{t+1} u_{t}+\Delta u^{2}-\delta \Delta u_{t} \\
=\mu(\cos t+\cos \sqrt{2} t)|u|^{\rho-1}(u \nabla \cdot u+u \Delta u), \\
x \in \Omega, \quad t \geq 0, \\
u=\Delta u=0, \quad x \in \partial \Omega, \quad t>0, \\
u(0, x)=u_{0}(x), \quad u_{t}(0, x)=v_{0}(x), \quad x \in \Omega .
\end{gathered}
$$

If $|\mu|+|\nu|$ is small enough by Proposition 21, problem (94) has an asymptotically almost-periodic mild solution. In fact, take $h(u)=g(u)=u|u|^{\rho-1}, a(t)=\cos t+\cos \sqrt{2} t$, and $b(t)=$ $1 /(t+1)$.

The next application is a modification of problem (79). Let $k$ be a fixed nonnegative integer and let $c: \mathbb{R} \rightarrow \mathbb{R}^{+}$and $b: \mathbb{R}^{+} \rightarrow \mathbb{R}$ be two bounded continuous functions. Suppose that $f \in A A P\left(\mathbb{R}^{+} \times \mathbb{R}^{N} \times \mathbb{R} \times \mathbb{R}, \mathbb{R}\right)$ satisfies the following growth condition:

$$
\begin{aligned}
& |f(t, x, u, w)-f(t, x, \widetilde{u}, \widetilde{w})| \\
& \quad \leq c(t)\left[|w-\widetilde{w}|+|u-\widetilde{u}|\left(1+|u|^{k}+|\widetilde{u}|^{k}\right)\right],
\end{aligned}
$$

for each $t \geq 0, x \in \mathbb{R}^{N}, u, \widetilde{u}, w, \widetilde{w} \in \mathbb{R}$. We consider the following partial differential equation

$$
\begin{gathered}
u_{t t}+b(t) u_{t}+\Delta^{2} u-\delta \Delta u_{t}=f(t, x, u, \nabla \cdot u), \\
x \in \Omega, \quad t \geq 0, \\
u=\Delta u=0, \quad x \in \partial \Omega, \quad t>0, \\
u(0, x)=u_{0}(x), \quad u_{t}(0, x)=v_{0}(x), \quad x \in \Omega .
\end{gathered}
$$

We model (96) in the abstract form (1) in a similar way to problem (79). That is we set $\eta=\delta / 2, p>N$ (hence $H_{p}^{2}(\Omega) \hookrightarrow C^{1}(\Omega)$ which will be useful to get some a priori estimates) and we consider the operator $A$ as defined in (81) and (82). Suppose that

$$
\sup _{t \geq 0}\left(\int_{\Omega}|f(t, x, 0,0)|^{p} d x\right)^{1 / p}<\infty .
$$

We define $\widetilde{F}: \mathbb{R}^{+} \times\left[L^{p}(\Omega)\right]^{1 / 2} \times L^{p}(\Omega) \rightarrow L^{p}(\Omega)$ by

$$
\widetilde{F}\left(t, \varphi_{1}, \varphi_{2}\right)(x)=f\left(t, x, \varphi_{1}(x), \nabla \cdot \varphi_{1}(x)\right)-b(t) \varphi_{2}(x) .
$$

We observe that $\widetilde{F}$ is well defined. In fact, we get the following estimates:

$$
\begin{aligned}
& \left(\int_{\Omega}\left|f\left(t, x, \varphi_{1}(x), \nabla \cdot \varphi_{1}(x)\right)\right|^{p} d x\right)^{1 / p} \\
& \leq c(t)\left(\left(\int_{\Omega}\left|\nabla \cdot \varphi_{1}(x)\right|^{p} d x\right)^{1 / p}\right. \\
& +\left(\int_{\Omega}\left|\varphi_{1}(x)\right|^{p}\left(1+\left|\varphi_{1}(x)\right|^{k}\right)^{p} d x\right)^{1 / p} \\
& \left.+\left(\int_{\Omega}|f(t, x, 0,0)|^{p} d x\right)^{1 / p}\right)^{1 / p} \\
& \leq\|c\|_{\infty}\left(\left\|\nabla \cdot \varphi_{1}\right\|_{L^{p}(\Omega)}+\left(1+\left\|\varphi_{1}\right\|_{L^{\infty}(\Omega)}^{k}\right)\left\|\varphi_{1}\right\|_{L^{p}(\Omega)}\right. \\
& \left.+\left(\int_{\Omega}|f(t, x, 0,0)|^{p} d x\right)^{1 / p}\right)
\end{aligned}
$$

On the other hand, for $\varphi_{1}, \widetilde{\varphi}_{1} \in\left[L^{p}(\Omega)\right]^{1 / 2}$ and $\varphi_{2}, \widetilde{\varphi}_{2} \in L^{p}(\Omega)$ we have the following estimate:

$$
\begin{aligned}
& \left\|\widetilde{F}\left(t, \varphi_{1}, \varphi_{2}\right)-\widetilde{F}\left(t, \widetilde{\varphi}_{1}, \widetilde{\varphi}_{2}\right)\right\|_{L^{p}(\Omega)} \\
& \leq 4 \max \left\{\|c\|_{\infty},\|b\|_{\infty}\right\}\left(1+\left\|\varphi_{1}\right\|_{H_{p}^{2}(\Omega)}^{k}+\left\|\widetilde{\varphi}_{1}\right\|_{H_{p}^{2}(\Omega)}^{k}\right) \\
& \quad \times\left(\left\|\varphi_{1}-\widetilde{\varphi}_{1}\right\|_{H_{p}^{2}(\Omega)}+\left\|\varphi_{2}-\widetilde{\varphi}_{2}\right\|_{L_{p}(\Omega)}\right),
\end{aligned}
$$

which means that $\widetilde{F}$ satisfies (42) with $L_{\widetilde{F}}(r)=$ $4 \max \left\{\|c\|_{\infty},\|b\|_{\infty}\right\}\left(1+2 r^{k}\right)$.

Since $f \in A A P\left(\mathbb{R}^{+} \times \mathbb{R}^{N} \times \mathbb{R} \times \mathbb{R}, \mathbb{R}\right)$, there are two functions

$$
\begin{aligned}
& f_{a p} \in A P\left(\mathbb{R} \times \mathbb{R}^{N} \times \mathbb{R} \times \mathbb{R}, \mathbb{R}\right), \\
& \varphi_{f} \in C_{0}\left(\mathbb{R}^{+} \times \mathbb{R}^{N} \times \mathbb{R} \times \mathbb{R}, \mathbb{R}\right),
\end{aligned}
$$

so that $f=f_{a p}+\varphi_{f}$. Let $K$ be a compact subset of $\left[L^{p}(\Omega)\right]^{1 / 2} \times$ $L^{p}(\Omega)$. For $\varphi_{1} \in\left[L^{p}(\Omega)\right]^{1 / 2}$ and $\varphi_{2} \in L^{p}(\Omega)$, we set

$$
\begin{gathered}
\widetilde{F}_{a p}\left(t, \varphi_{1}, \varphi_{2}\right)(x)=f_{a p}\left(t, x, \varphi_{1}(x), \nabla \cdot \varphi_{1}(x)\right), \\
\widetilde{\Phi}_{\widetilde{F}}\left(t, \varphi_{1}, \varphi_{2}\right)(x)=\varphi_{f}\left(t, x, \varphi_{1}(x), \nabla \cdot \varphi_{1}(x)\right)-b(t) \varphi_{2}(x) .
\end{gathered}
$$

If $\left(\varphi_{1}, \varphi_{2}\right) \in K$, then

$$
\begin{aligned}
& \left\|\widetilde{\Phi}_{\widetilde{F}}\left(t, \varphi_{1}, \varphi_{2}\right)\right\|_{L^{p}(\Omega)} \\
& \quad \leq\left(\int_{\Omega}\left|\varphi_{f}\left(t, x, \varphi_{1}(x), \nabla \cdot \varphi_{1}(x)\right)\right|^{p} d x\right)^{1 / p} \\
& \quad+|b(t)| M_{K},
\end{aligned}
$$

where $M_{K}$ is given by (88). We note that using Sobolev 
embedding we have that there is a positive constant $\widetilde{c}>0$ depending only on $K$ so that

$$
\sup _{x \in \Omega}\left|\varphi_{1}(x)\right| \leq \widetilde{c}, \quad \sup _{x \in \Omega}\left|\nabla \cdot \varphi_{1}(x)\right| \leq \widetilde{c} .
$$

Taking into account the fact that $Y=\bar{\Omega} \times B_{\widetilde{c}}\left(\mathbb{R}^{2}\right)$ is a compact subset of $\mathbb{R}^{N+2},(4.29)$ and (103). It follows that $\lim _{t \rightarrow \infty} \widetilde{\Phi}_{\widetilde{F}}\left(t, \varphi_{1}, \varphi_{2}\right)=0$ uniformly for $\left(\varphi_{1}, \varphi_{2}\right) \in K$. Hence,

$$
\widetilde{\Phi}_{\widetilde{F}} \in C_{0}\left(\mathbb{R}^{+} \times\left[L^{p}(\Omega)\right]^{1 / 2} \times L^{p}(\Omega), L^{p}(\Omega)\right) .
$$

As an immediate consequence of Definition 3 and using the fact that $\Omega$ is bounded we have that

$$
\widetilde{F}_{a p} \in A P\left(\mathbb{R} \times\left[L^{p}(\Omega)\right]^{1 / 2} \times L^{p}(\Omega), L^{p}(\Omega)\right) .
$$

Since $\widetilde{F}=\widetilde{F}_{a p}+\widetilde{\Phi}_{\widetilde{F}}$, we conclude that

$$
\widetilde{F} \in A A P\left(\mathbb{R}^{+} \times\left[L^{p}(\Omega)\right]^{1 / 2} \times L^{p}(\Omega), L^{p}(\Omega)\right) .
$$

Proposition 23. Under the previous conditions, if $\max \left\{\|c\|_{\infty},\|b\|_{\infty}\right\}$ is small enough, then problem (96) has an asymptotically almost-periodic mild solution.

Proof. We argue as follows. Let us choose $r>0$ such that $K / r C \sup _{t \geq 0}\left(\int_{\Omega}|f(t, x, 0,0)|^{p} d x\right)^{1 / p}$ is small enough, where $K$ and $C$ are constants given in (7). Then, condition (47) is fulfilled. Now applying Theorem 17 we conclude the proof.

Let $a: \mathbb{R}^{+} \rightarrow \mathbb{R}$ be a bounded continuous function and let $\beta$ be in $(0,1)$ and $\delta>0$. In a bounded smooth domain $\Omega \subseteq$ $\mathbb{R}^{N}$, we consider the following partial differential equation:

$$
\begin{gathered}
u_{t t}+\Delta^{2} u-\delta \Delta u_{t}=a(t)\left|\int_{\Omega} \nabla \cdot u(t, x) d x\right|^{\beta} \Phi_{0}, \\
x \in \Omega, \quad t \geq 0, \\
u=\Delta u=0, \quad x \in \partial \Omega, \quad t>0, \\
u(0, x)=u_{0}(x), \quad u_{t}(0, x)=v_{0}(x), \quad x \in \Omega,
\end{gathered}
$$

where $\Phi_{0} \in L^{p}(\Omega), p>N / 2$. We model (108) in the abstract form (1); that is, we set $\eta=\delta / 2$ and we consider the operator $A$ as in (81) and (82). We define $f: \mathbb{R}^{+} \times\left[L^{p}(\Omega)\right]^{1 / 2} \times L^{p}(\Omega) \rightarrow$ $L^{p}(\Omega)$ by

$$
f\left(t, \varphi_{1}, \varphi_{2}\right)(x)=a(t)\left|\int_{\Omega} \nabla \cdot \varphi_{1}(\xi) d \xi\right|^{\beta} \Phi_{0}(x) .
$$

To establish our next result, we assume the following conditions.

(H1) Let $a(\cdot)=a_{a p}(\cdot)+\varphi_{a}(\cdot)$ be in $A A P\left(\mathbb{R}^{+}\right)$, with $a_{a p}(\cdot) \epsilon$ $A P(\mathbb{R})$ and $\varphi_{a}(\cdot) \in C_{0}\left(\mathbb{R}^{+}\right)$.
(H2) There is a continuous nondecreasing function $h$ : $[0, \infty) \rightarrow[1, \infty)$ such that $h(t) \rightarrow \infty$ as $t \rightarrow \infty$ and

$$
\begin{aligned}
& \lim _{t \rightarrow \infty} \frac{1}{h(t)} \int_{0}^{t} e^{-C(t-s)} h(s)^{\beta} d s=0, \\
& \sup _{t \geq 0} \int_{0}^{t} e^{-C(t-s)}|a(t)| h(s)^{\beta} d s<\infty .
\end{aligned}
$$

Proposition 24. Under the previous conditions, problem (108) has an asymptotically almost-periodic mild solution.

Proof. Let $q>0$ be given so that $(1 / q)+(1 / p)=1$. We can infer the following estimate:

$$
\left\|f\left(t, \varphi_{1}, \varphi_{2}\right)\right\|_{L^{p}(\Omega)} \leq\|a\|_{\infty}\left\|\Phi_{0}\right\|_{L^{p}(\Omega)}|\Omega|^{\beta / q}\left\|\varphi_{1}\right\|_{H_{p}^{2}(\Omega)}^{\beta} .
$$

Hence, we can define $W_{f}$ in $(W)$ by $W_{f}(\xi)=$ $\|a\|_{\infty}\left\|\Phi_{0}\right\|_{L^{p}(\Omega)}|\Omega|^{\beta / q} \xi^{\beta}$. Let $K$ be a compact subset of $\left[L^{p}(\Omega)\right]^{1 / 2} \times L^{p}(\Omega)$ and let $M_{K}$ be as in (88). We set

$$
\begin{gathered}
f_{a p}\left(t, \varphi_{1}, \varphi_{2}\right)(x)=a_{a p}(t)\left|\int_{\Omega} \nabla \cdot \varphi_{1}(\xi) d \xi\right|^{\beta} \Phi_{0}(x), \\
\Phi_{f}^{\#}\left(t, \varphi_{1}, \varphi_{2}\right)(x)=\varphi_{a}(t)\left|\int_{\Omega} \nabla \cdot \varphi_{1}(\xi) d \xi\right|^{\beta} \Phi_{0}(x), \\
{\left[\begin{array}{l}
\varphi_{1} \\
\varphi_{2}
\end{array}\right],\left[\begin{array}{l}
\psi_{1} \\
\psi_{2}
\end{array}\right] \in K .}
\end{gathered}
$$

We have the following estimates:

$$
\begin{gathered}
\left\|f_{a p}\left(t+\tau, \varphi_{1}, \varphi_{2}\right)-f_{a p}\left(t, \varphi_{1}, \varphi_{2}\right)\right\|_{L^{p}(\Omega)} \\
\leq|\Omega|^{\beta / q} M_{K}^{\beta}\left\|\Phi_{0}\right\|_{L^{p}(\Omega)}\left|a_{a p}(t+\tau)-a_{a p}(t)\right|, \\
\left\|\Phi_{f}^{\#}\left(t, \varphi_{1}, \varphi_{2}\right)\right\|_{L^{p}(\Omega)} \leq|\Omega|^{\beta / q} M_{K}^{\beta}\left\|\Phi_{0}\right\|_{L^{p}(\Omega)}\left|\varphi_{a}(t)\right|, \\
\left\|f\left(t, \varphi_{1}, \varphi_{2}\right)-f\left(t, \psi_{1}, \psi_{2}\right)\right\|_{L^{p}(\Omega)} \\
\leq|\Omega|^{\beta / q}\|a\|_{\infty}\left\|\Phi_{0}\right\|_{L^{p}(\Omega)}\left\|\varphi_{1}-\psi_{1}\right\|_{H_{p}^{2}(\Omega)}^{\beta} .
\end{gathered}
$$

It follows from (113) and (114) that $f \in A A P\left(\mathbb{R}^{+} \times\left[L^{p}(\Omega)\right]^{1 / 2} \times\right.$ $\left.L^{p}(\Omega), L^{p}(\Omega)\right)$. In addition, from the estimate (115), we obtain that $f$ is uniformly continuous on compact sets. 
From $\left(\mathrm{H}_{2}\right)$, for $\left[\begin{array}{c}u_{i} \\ v_{i}\end{array}\right] \in C_{h}\left(\left[L^{p}(\Omega)\right]^{1 / 2} \times L^{p}(\Omega)\right), i=1,2$, we can infer that

$$
\begin{aligned}
\frac{1}{h(t)} \int_{0}^{t} e^{-C(t-s)} W_{f}(L h(s)) d s & \\
=\|a\|_{\infty}\left\|\Phi_{0}\right\|_{L^{p}(\Omega)}|\Omega|^{\beta / q} L^{\beta}\left(\frac{1}{h(t)} \int_{0}^{t} e^{-C(t-s)} h(s)^{\beta} d s\right) & \longrightarrow 0, \\
t & \longrightarrow \infty,
\end{aligned}
$$

$$
\begin{aligned}
\int_{0}^{t} e^{-C(t-s)}\left\|f\left(s, u_{1}(s), v_{1}(s)\right)-f\left(s, u_{2}(s), v_{2}(s)\right)\right\|_{L^{p}(\Omega)} d s \\
\leq|\Omega|^{\beta / q}\left\|\Phi_{0}\right\|_{L^{p}(\Omega)} \int_{0}^{t} e^{-C(t-s)}|a(s)|\left\|u_{1}(s)-u_{2}(s)\right\|_{H_{p}^{2}(\Omega)}^{\beta} d s \\
=|\Omega|^{\beta / q}\left\|\Phi_{0}\right\|_{L^{p}(\Omega)} \int_{0}^{t} e^{-C(t-s)}|a(s)| h(s)^{\beta} \\
\quad \times\left(\frac{\left\|u_{1}(s)-u_{2}(s)\right\|_{H_{p}^{2}(\Omega)}}{h(s)}\right)^{\beta} d s \\
\leq|\Omega|^{\beta / q}\left\|\Phi_{0}\right\|_{L^{p}(\Omega)} \sup _{t \geq 0}\left(\int_{0}^{t} e^{-C(t-s)}|a(t)| h(s)^{\beta} d s\right) \\
\quad \times\left[\operatorname { s u p } _ { t \geq 0 } \left(\frac { 1 } { h ( t ) } \left[\left\|u_{1}(t)-u_{2}(t)\right\|_{H_{p}^{2}(\Omega)}\right.\right.\right. \\
\left.\left.\left.+\left\|v_{1}(t)-v_{2}(t)\right\|_{L^{p}(\Omega)}\right]\right)\right]^{\beta} .
\end{aligned}
$$

Therefore, conditions (c) and (d) of Theorem 19 are satisfied. A straightforward computation shows that (e) holds. We can prove that the set $\left\{f\left(t, \varphi_{1}, \varphi_{2}\right): 0 \leq t \leq a,\left\|\varphi_{1}\right\|_{H_{p}^{2}(\Omega)}+\right.$ $\left.\left\|\varphi_{2}\right\|_{L^{p}(\Omega)} \leq r\right\}$ is relatively compact in $L^{p}(\Omega)$. In fact, first, we denote by $\tilde{u}$ the zero extension of $u$ outside $\Omega$; that is,

$$
\widetilde{u}(x)= \begin{cases}u(x) & \text { if } x \in \Omega, \\ 0 & \text { if } x \in \mathbb{R}^{N} \backslash \Omega .\end{cases}
$$

For every number $\varepsilon>0$, there exists a number $\delta>0$ and a subset $G \subset \subset \Omega$ such that for every $\varrho \in \mathbb{R}^{N}$ with $|\varrho|<\delta$

$$
\begin{gathered}
\int_{\Omega}\left|\widetilde{\Phi}_{0}(x+\varrho)-\widetilde{\Phi}_{0}(x)\right|^{p} d x<\left(\frac{\varepsilon}{r^{\beta}\|a\|_{\infty}|\Omega|^{\beta / q}}\right)^{p}, \\
\int_{\Omega \backslash G}\left|\Phi_{0}(x)\right|^{p} d x<\left(\frac{\varepsilon}{r^{\beta}\|a\|_{\infty}|\Omega|^{\beta / q}}\right)^{p} .
\end{gathered}
$$

Hence,

$$
\begin{aligned}
& \int_{\Omega}\left|\tilde{f}\left(t, \varphi_{1}, \varphi_{2}\right)(x+\varrho)-\widetilde{f}\left(t, \varphi_{1}, \varphi_{2}\right)(x)\right|^{p} d x \\
& \leq\left[r^{\beta}\|a\|_{\infty}|\Omega|^{\beta / q}\right]^{p} \times \int_{\Omega}\left|\widetilde{\Phi}_{0}(x+\rho)-\widetilde{\Phi}_{0}(x)\right|^{p} d x \leq \varepsilon, \\
& \int_{\Omega \backslash G}\left|f\left(t, \varphi_{1}, \varphi_{2}\right)(x)\right|^{p} d x \leq\left[r^{\beta}\|a\|_{\infty}|\Omega|^{\beta / q}\right]^{p} \\
& \quad \times \int_{\Omega \backslash G}\left|\Phi_{0}(x)\right|^{p} d x \leq \varepsilon .
\end{aligned}
$$

From [38, Theorem 2.21] the desired assertion follows. Note finally that using Remark 20 we have that (108) has an asymptotically almost periodic mild solution. This ends the proof.

\section{Acknowledgments}

This work was written while the first author was visiting the Universidad de La Frontera (Temuco, Chile). He is grateful to the Applicable Analysis Group and the Department of Mathematics and Statistics, for its generous hospitality and providing a stimulating atmosphere to work. C. Cuevas has been partially supported by Programa Atracción e Inserción (PAI-MEC) Grant 80112008 (CONICYT-CHILE). C. Lizama has been partially supported by Project Anillo ACT 79 and $\mathrm{H}$. Soto has been partially supported by DIUFRO DI 12-0002.

\section{References}

[1] D. Henry, Geometric Theory of Semilinear Parabolic Equations, vol. 840 of Lecture Notes in Mathematics, Springer, Berlin, Germany, 1981.

[2] A. N. Carvalho and J. W. Cholewa, "Attractors for strongly damped wave equations with critical nonlinearities," Pacific Journal of Mathematics, vol. 207, no. 2, pp. 287-310, 2002.

[3] S. P. Chen and R. Triggiani, "Proof of extensions of two conjectures on structural damping for elastic systems," Pacific Journal of Mathematics, vol. 136, no. 1, pp. 15-55, 1989.

[4] S. P. Chen and R. Triggiani, "Characterization of domains of fractional powers of certain operators arising in elastic systems, and applications," Journal of Differential Equations, vol. 88, no. 2, pp. 279-293, 1990.

[5] S. P. Chen and R. Triggiani, "Proof of two conjectures by G. Chen and D. L. Russell on structural damping for elastic systems," in Approximation and Optimization, vol. 1354 of Lecture Notes in Mathematics, pp. 234-256, Springer, 1988.

[6] D. Bahuguna, "Strongly damped semilinear equations," Journal of Applied Mathematics and Stochastic Analysis, vol. 8, no. 4, pp. 397-404, 1995.

[7] V. Georgiev and G. Todorova, "Existence of a solution of the wave equation with nonlinear damping and source terms," Journal of Differential Equations, vol. 109, no. 2, pp. 295-308, 1994.

[8] F. Gazzola and M. Squassina, "Global solutions and finite time blow up for damped semilinear wave equations," Annales de l'Institut Henri Poincaré, vol. 23, no. 2, pp. 185-207, 2006. 
[9] J. M. Ghidaglia and A. Marzocchi, "Longtime behaviour of strongly damped wave equations, global attractors and their dimension," SIAM Journal on Mathematical Analysis, vol. 22, no. 4, pp. 879-895, 1991.

[10] P. Massatt, "Limiting behavior for strongly damped nonlinear wave equations," Journal of Differential Equations, vol. 48, no. 3 , pp. 334-349, 1983.

[11] G. F. Webb, "Existence and asymptotic behavior for a strongly damped nonlinear wave equation," Canadian Journal of Mathematics, vol. 32, no. 3, pp. 631-643, 1980.

[12] S. Borini and V. Pata, "Uniform attractors for a strongly damped wave equation with linear memory," Asymptotic Analysis, vol. 20, no. 3-4, pp. 263-277, 1999.

[13] S. M. Bruschi, A. N. Carvalho, J. W. Cholewa, and T. Dlotko, "Uniform exponential dichotomy and continuity of attractors for singularly perturbed damped wave equations," Journal of Dynamics and Differential Equations, vol. 18, no. 3, pp. 767-814, 2006.

[14] A. N. Carvalho and J. W. Cholewa, "Strongly damped wave equations in $W_{0}^{1, p}(\Omega) \times L^{p}(\Omega)$," Discrete and Continuous Dynamical Systems, pp. 230-239, 2007.

[15] H. Li, S. Zhou, and F. Yin, "Global periodic attractor for strongly damped wave equations with time-periodic driving force," Journal of Mathematical Physics, vol. 45, no. 9, pp. 34623467, 2004.

[16] V. Pata and M. Squassina, "On the strongly damped wave equation," Communications in Mathematical Physics, vol. 253, no. 3, pp. 511-533, 2005.

[17] A. N. Carvalho and J. W. Cholewa, "Local well posedness for strongly damped wave equations with critical nonlinearities," Bulletin of the Australian Mathematical Society, vol. 66, no. 3, pp. 443-463, 2002.

[18] R. Ikehata, "Decay estimates of solutions for the wave equations with strong damping terms in unbounded domains," Mathematical Methods in the Applied Sciences, vol. 24, no. 9, pp. 659670, 2001.

[19] G. Avalos and I. Lasiecka, "Optimal blowup rates for mininal energy null control for the structurally damped wave equation," Annali della Scuola Normale Superiore di Pisa, vol. 2, pp. 601616, 2003.

[20] S. A. Messaoudi, "Blow up in a nonlinearly damped wave equation," Mathematische Nachrichten, vol. 231, pp. 105-111, 2001.

[21] A. N. Carvalho, J. W. Cholewa, and T. Dlotko, "Strongly damped wave problems: bootstrapping and regularity of solutions," Journal of Differential Equations, vol. 244, no. 9, pp. 2310-2333, 2008.

[22] R. P. Agarwal, B. de Andrade, and C. Cuevas, "On type of periodicity and ergodicity to a class of fractional order differential equations," Advances in Difference Equations, vol. 2010, Article ID 179750, 25 pages, 2010.

[23] R. P. Agarwal, B. de Andrade, and C. Cuevas, "Weighted pseudo-almost periodic solutions of a class of semilinear fractional differential equations," Nonlinear Analysis, vol. 11, no. 5, pp. 3532-3554, 2010.

[24] A. Berger, S. Siegmund, and Y. Yi, "On almost automorphic dynamics in symbolic lattices," Ergodic Theory and Dynamical Systems, vol. 24, no. 3, pp. 677-696, 2004.

[25] C. Cuevas and M. Pinto, "Existence and uniqueness of pseudo almost periodic solutions of semilinear Cauchy problems with non dense domain," Nonlinear Analysis A, vol. 45, no. 1, pp. 7383, 2001.
[26] B. de Andrade, C. Cuevas, and E. Henríquez, "Asymptotic periodicity and almost automorphy for a class of Volterra integro-differential equations," Mathematical Methods in the Applied Sciences, vol. 35, no. 7, pp. 795-811, 2012.

[27] T. Diagana, G. Nguerekata, and N. V. Minh, "Almost automorphic solutions of evolution equations," Proceedings of the American Mathematical Society, vol. 132, no. 11, pp. 3289-3298, 2004.

[28] H. Henríquez and C. Cuevas, "Almost automorphy for abstract neutral differential equations via control theory," Annali di Matematica Pura ed Applicata, vol. 192, no. 3, pp. 393-405, 2013.

[29] J. Kaczorowski and O. Ramaré, "Almost periodicity of some error terms in prime number theory," Acta Arithmetica, vol. 106, no. 3, pp. 277-297, 2003.

[30] J. H. Liu and X. Q. Song, "Almost automorphic and weighted pseudo almost automorphic solutions of semilinear evolution equations," Journal of Functional Analysis, vol. 258, no. 1, pp. 196-207, 2010.

[31] N. V. Minh, T. Naito, and G. Nguerekata, "A spectral countability condition for almost automorphy of solutions of differential equations," Proceedings of the American Mathematical Society, vol. 134, no. 11, pp. 3257-3266, 2006.

[32] W. Shen and Y. Yi, "Almost automorphic and almost periodic dynamics in skew-product semi flows," Memoirs of the American Mathematical Society, vol. 136, no. 647, 1998.

[33] S. Zaidman, Almost-Periodic Functions in Abstract Spaces, vol. 126 of Research Notes in Mathematics, Pitman, Boston, Mass, USA, 1985.

[34] A. M. Fink, Almost Periodic Differential Equations, vol. 377 of Lecture Notes in Mathematics, Springer, Berlin, Germany, 1974.

[35] S. Zaidman, "Solutions of almost-periodic abstract differential equations with relatively compact range," Nonlinear Analysis, vol. 8, no. 9, pp. 1091-1093, 1984.

[36] G. Gripenberg, S. O. Londen, and O. Staffans, Volterra Integral and Functional Equations, vol. 34, Cambridge University Press, Cambridge, UK, 1990.

[37] R. Temam, Infinite-Dimensional Dynamical Systems in Mechanics and Physics, vol. 68, Springer, New York, NY, USA, 2nd edition, 1997.

[38] R. A. Adams, Sobolev Spaces, Academic Press, London, UK, 1975. 


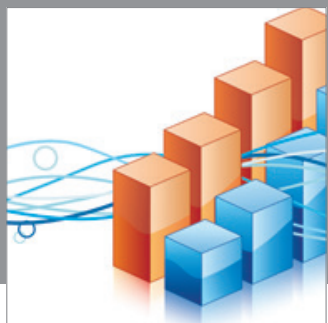

Advances in

Operations Research

mansans

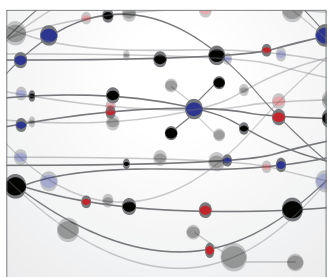

The Scientific World Journal
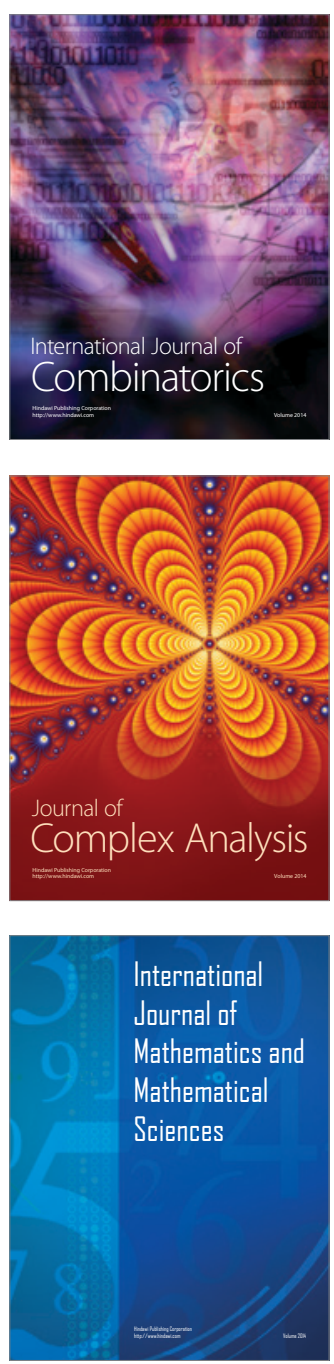
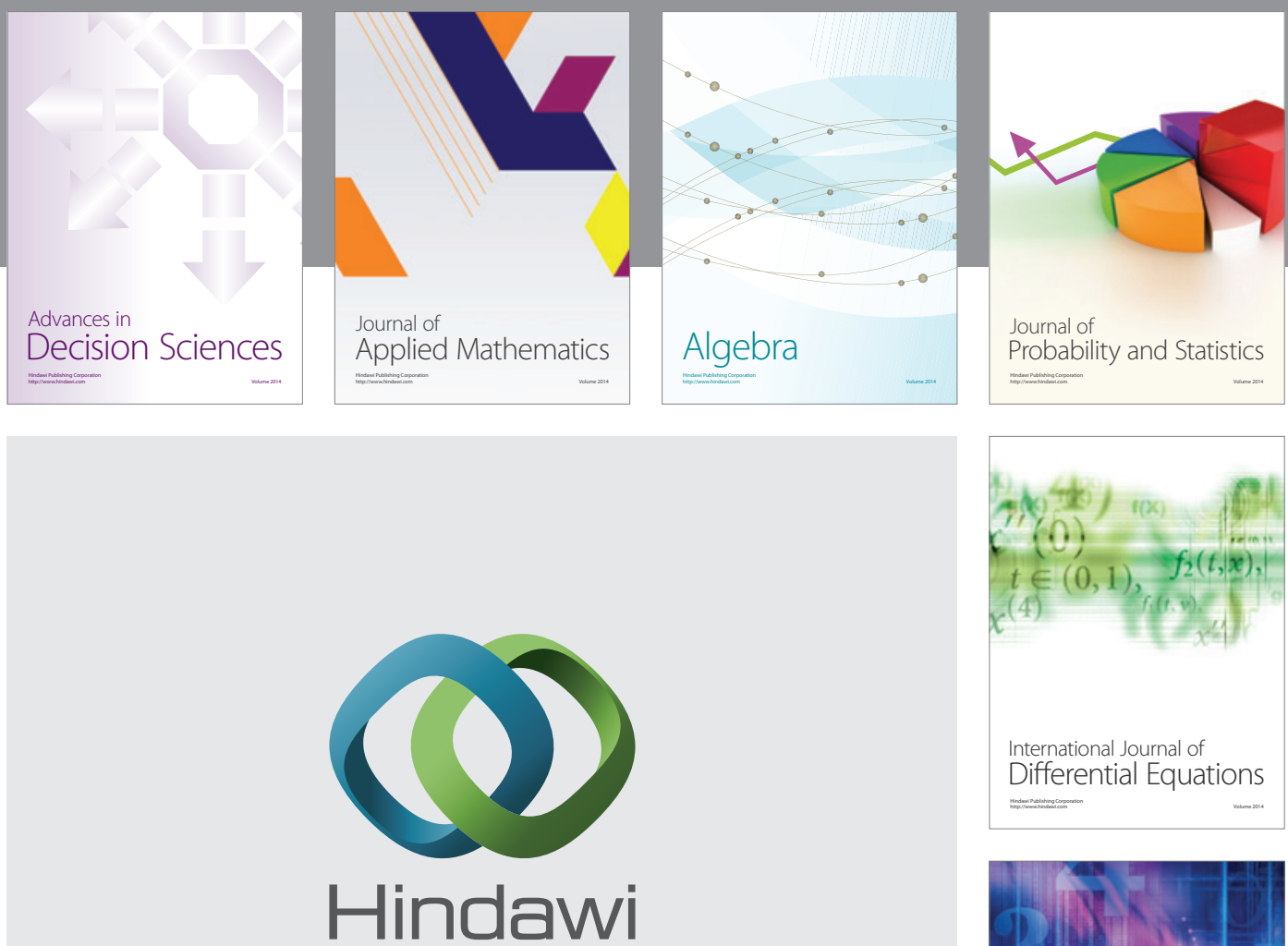

Submit your manuscripts at http://www.hindawi.com
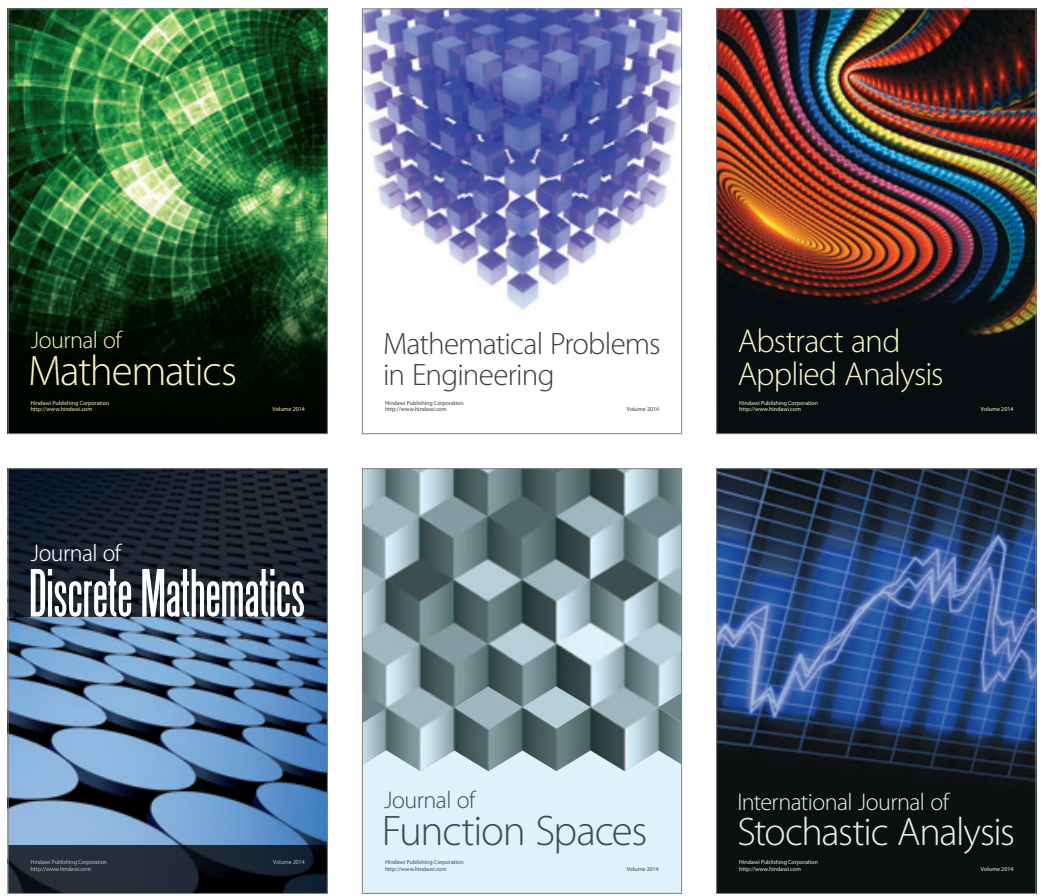

Journal of

Function Spaces

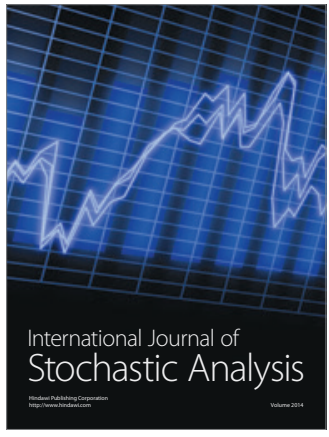

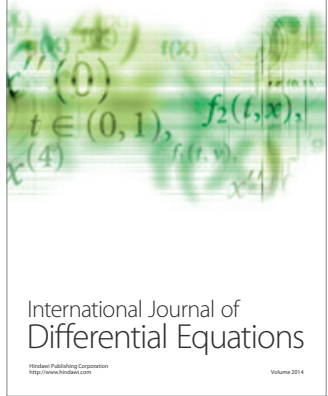
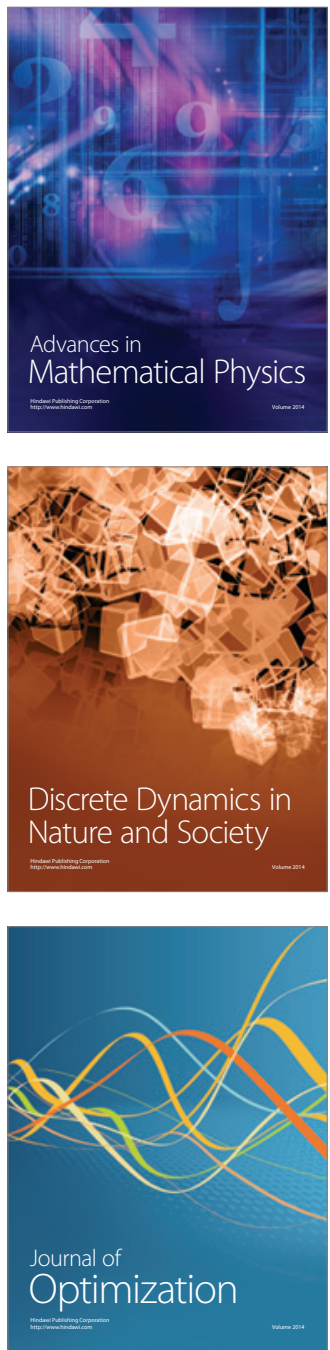